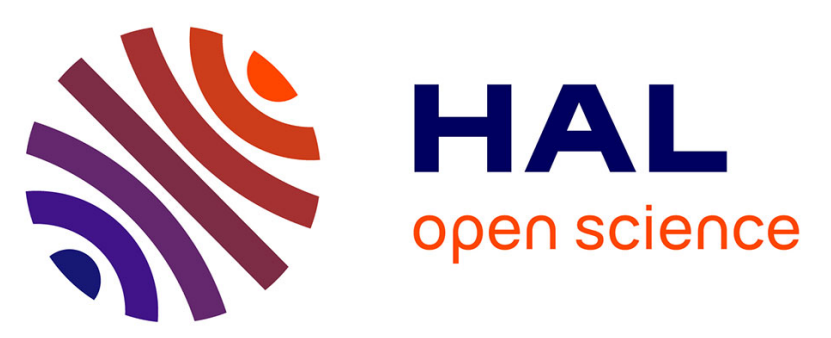

\title{
The effects of low-input grazing systems and milk pasteurisation on the chemical composition, microbial communities, and sensory properties of uncooked pressed cheeses
}

Marie Fretin, Anne Ferlay, Isabelle Verdier-Metz, Florence Fournier, Marie-Christine Montel, Anne Farruggia, Céline Delbes, Bruno Martin

\section{To cite this version:}

Marie Fretin, Anne Ferlay, Isabelle Verdier-Metz, Florence Fournier, Marie-Christine Montel, et al.. The effects of low-input grazing systems and milk pasteurisation on the chemical composition, microbial communities, and sensory properties of uncooked pressed cheeses. International Dairy Journal, 2017, 64, pp.56-67. 10.1016/j.idairyj.2016.09.007 . hal-01607900

\section{HAL Id: hal-01607900 https://hal.science/hal-01607900}

Submitted on 27 May 2020

HAL is a multi-disciplinary open access archive for the deposit and dissemination of scientific research documents, whether they are published or not. The documents may come from teaching and research institutions in France or abroad, or from public or private research centers.
L'archive ouverte pluridisciplinaire HAL, est destinée au dépôt et à la diffusion de documents scientifiques de niveau recherche, publiés ou non, émanant des établissements d'enseignement et de recherche français ou étrangers, des laboratoires publics ou privés.

\section{다(1)(2)}

Distributed under a Creative Commons Attribution - ShareAlikel 4.0 International 


\title{
The effects of low-input grazing systems and milk pasteurisation on the chemical composition, microbial communities, and sensory properties of uncooked pressed cheeses
}

\author{
Marie Frétin ${ }^{\mathrm{a}, \mathrm{b}}$, Anne Ferlay ${ }^{\mathrm{b}}$, Isabelle Verdier-Metz ${ }^{\mathrm{a}}$, Florence Fournier ${ }^{\mathrm{c}}$, \\ Marie-Christine Montel ${ }^{a}$, Anne Farruggia ${ }^{\mathrm{b}}$, Céline Delbès ${ }^{\mathrm{a}, ~}{ }^{*}$, Bruno Martin ${ }^{\mathrm{b}}$ \\ a INRA, UR545 Fromagères, Côte de Reyne, F-15000 Aurillac, France \\ ${ }^{\mathrm{b}}$ INRA, UMR1213 Herbivores, VetAgro Sup, F-63122 Saint-Genès-Champanelle, France \\ ${ }^{\mathrm{c}}$ INRA, UE1414 Herbipôle, F-63122 Saint-Genès-Champanelle, France
}

\section{A R T I C L E I N F O}

\section{Article history:}

Received 8 June 2016

Received in revised form

7 September 2016

Accepted 13 September 2016

Available online 8 October 2016

\begin{abstract}
A B S T R A C T
The objective of this work was to determine whether the effects of farming systems on the cheese sensory properties are reduced when the milk used to make cheese is pasteurised. The milk of cows reared under two grazing systems (extensive and semi-extensive) was processed into raw and pasteurised milk cheeses. These grazing systems were mainly differentiable by the milk fatty acid (FA) composition and, to a lesser extent, by their levels of lactic acid bacteria, which were higher in milk from the extensive grazing system. In cheese, the different FA composition persisted and affected the texture of the cheese regardless of the milk treatment. The effects of each grazing system were indistinguishable in several cheese characteristics (proteolysis, texture, and aroma) after pasteurisation. In contrast, among pasteurised milk cheeses, those resulting from an extensive system had greyer rinds, while no difference in rind colour was observed among raw milk cheeses.
\end{abstract}

() 2016 Elsevier Ltd. All rights reserved.

\section{Introduction}

The sensory quality of cheeses depends on many factors linked to cheese-making technology and to the chemical and microbial characteristics of raw milk. Farming practices that modify the characteristics of raw milk are important to consider, especially when milk is processed after minimal treatment, as in most farmhouse and quality-labelled cheeses granted a geographical indication. Particular attention has been focused on animal feed, which varies widely according to geographic region and is therefore related to the concept of "terroir" (Coulon, Delacroix-Buchet, Martin, \& Pirisi, 2004). The effects of the type of forage distributed to animals (e.g., pasture, hay, grass silage, or maize silage) on the sensory properties of cheese are well documented (Martin, VerdierMetz, Buchin, Hurtaud, \& Coulon, 2005). These effects are linked to changes in milk characteristics, and particular attention has been paid to milk fat, which is known to vary widely with different animal feeds (Chilliard et al., 2007; Collomb, Bütikofer, Siber, Jeangros,

\footnotetext{
* Corresponding author. Tel.: +33471456419.

E-mail address: celine.delbes@clermont.inra.fr (C. Delbès).
}

\& Bosset, 2002; Elgersma, Tamminga, \& Ellen, 2006; Gómez-Cortés et al., 2009).

Grazing cows produce milk with a higher proportion of unsaturated fatty acids (FAs) and a lower melting point, which results in creamier cheeses, than that of cows receiving winter diets (Martin et al., 2005). The higher concentration of carotenoids in milk fat from grazing cows also explains the yellower colour of cheeses produced from this milk than those produced from the milk of cows fed hay-, grain- or maize silage-based diets (Martin et al., 2005; Nozière et al., 2006). The role of milk fat in the formation of volatile compounds by lipolysis and the oxidation of unsaturated FA has been proposed as a possible explanation for the more intense and diversified aromas of cheeses from grazing cows in comparison with those from cows fed winter diets (Bovolenta et al., 2014; Coppa et al., 2011a). On the pasture, grassland characteristics (phenological stage and botanical composition) and the supplementation of grazed grass with additional forages and concentrates modify the FA composition of milk (Bargo, Delahoy, Schroeder, \& Muller, 2006; Coppa et al., 2015; Farruggia et al., 2014). The FA composition of milk has also been proposed to explain variations in the sensory properties of cheese attributed to grazing systems 
(Coppa et al., 2011a) or to the botanical composition of pastures (Coulon et al., 2004).

Milk microbiota has received little attention when explaining the influence of animal feeding on the sensory properties of cheese, although a great deal of research has been conducted on raw milk cheeses. In unpasteurised cheese production, the raw milk microbiota plays a key role on the cheese flavour development; for instance, experimental Salers-type cheeses manufactured from the same pasteurised milk and inoculated alternately with three different microbiota differed widely in their sensory attributes (Callon, Berdagué, Dufour, \& Montel, 2005). The raw milk microbiota depends mainly on the microbiota composition of sources directly in contact with the milk, such as the cow's teat skin and dairy equipment (milking machines, milk lines, and tanks), and on indirect sources such as animal feeding and bedding, drinking and washing water, stable and milking parlour air, and the milker (Montel et al., 2014). Verdier-Metz et al. (2002) showed that the microbiota of teat skin varied according to animal feeding. Consequently, the raw milk microbiota could be modified, which may explain the influence of animal feeding on the sensory properties of cheeses. This hypothesis is reinforced because some observations suggest that the effects of animal feeding on the sensory properties of cheese are reduced when milk is pasteurised (Verdier-Metz et al., 2002). Indeed, the reduction of the microbial diversity and of the associated microbial activities in milk after undergoing thermal treatment is known to affect cheese flavour (Irlinger \& Mounier, 2009).

Animal feeding in general, and grass grazing in particular, could influence milk microbiota, and we can hypothesise that these changes, associated with changes in milk composition, and especially with FA composition, could modify the sensory characteristics of cheeses. Based on the literature, milk pasteurisation may hide the putative differences between grazing systems due to an equalisation of milk microbiota level (Giaccone et al., 2016; VerdierMetz et al., 2002). To test these hypotheses, we implemented an experiment using two herds reared separately for two years under two grazing systems. We evaluated the combined effect of grazing systems and milk pasteurisation on (i) chemical composition, including FA, (ii) levels of microbial groups in milks and cheeses, and (iii) the sensory properties of cheese.

\section{Materials and methods}

\subsection{Experimental design}

The experiment was conducted at the experimental farm of Marcenat (INRA, UE1296 Monts d'Auvergne, France), which is located in an upland mountainous grassland area in central France $\left(45^{\circ} 18^{\prime} 21^{\prime \prime} \mathrm{N}, 2^{\circ} 50^{\prime} 13^{\prime \prime} \mathrm{E}\right.$, altitude $1135-1215 \mathrm{~m}$, annual rainfall $1100 \mathrm{~mm}$ ). The study was conducted in 2012 during the 2nd year of a long-term research project assessing the interactions between management practices and animal performances at the farm level (Pires et al., 2015). A total of 24 Holstein and 24 Montbeliarde spring-calving dairy cows and their corresponding heifers were divided between two independent farmlets denominated extensive (EXT) and semi-extensive (SEMI). The EXT system consisted of 60.5 ha of highly diversified grasslands (61.1 species per paddock on average). The paddocks were grazed using a long rotational duration (4 grazing cycles of 51 days on average) managed at a low stocking rate ( 0.66 livestock unit (LU) ha ${ }^{-1} ; \mathrm{LU}=600 \mathrm{~kg}$ of body weight). No concentrate was added to the diet of cows. The SEMI system was composed of 29.2 ha of permanent and old temporary grasslands ( +10 years; 38.6 species per paddock on average). The paddocks were grazed using a rapid rotational duration (5 grazing cycles of 41 days on average) exploited at a moderate stocking rate
(1.09 $\mathrm{LU} \mathrm{ha}^{-1}$ ). Four kilogrammes per cow of a pelleted concentrate composed of $40 \%$ wheat, $30 \%$ barley, $25 \%$ maize, and $5 \%$ molasses were distributed daily to the SEMI cows in a collective feed trough, after the morning milking during the entire grazing period. The botanical composition of the two grazing systems is summarised in Supplementary Table S1.

Two periods $(\mathrm{P})$ of three dates each of bulk milk sampling and cheese-making were chosen to examine the effects of the two grazing systems at different phenological stages of grass and different growth cycles: three dates in July 2012 (the 5th, 10th, and 12th) corresponding to peak flowering in the EXT paddock, and three dates in September 2012 (the 6th, 11th, and 13th) when the flowering period had ended.

The milk used for cheese-making was produced by a total of 15 and 17 spring-calving cows selected from among the 24 cows grazed under the EXT and SEMI systems, respectively, so that the breed, parity and lactation stage of the EXT and SEMI animals were as similar as possible. The allotment of the dairy cows is given in Supplementary Table S1. The animals were milked with the same milking machine at 6:30 am and 4:30 pm. All cows received a sterile $85 \%$ glycerol solution post-dipped on the teats following each milking. Animal and paddock characteristics are presented in Supplementary Table S1.

\subsection{Cheese-making}

For each cheese-making date and for each grazing system, the bulk morning milk was pooled with the previous evening's milk and stored at $4{ }^{\circ} \mathrm{C}$. Half of this milk was pasteurised $\left(72{ }^{\circ} \mathrm{C}\right.$ for $20 \mathrm{~s}$; PierreRalisi, Italy) prior to cheese-making. During each cheesemaking date, four small-size Cantal-type cheeses (10 kg) were manufactured concomitantly from $110 \mathrm{~L}$ of EXT or SEMI raw $(\mathrm{R})$ or pasteurised (PA) full-fat milk in separate vats. Cheese-making was performed as described previously by Martin, Pomiès, Pradel, Verdier-Metz, and Rémond (2009), except that whole milk heated to $33{ }^{\circ} \mathrm{C}$ was inoculated with $0.05 \mathrm{~g} 100 \mathrm{~kg}^{-1}$ of a mesophilic starter culture (Flora Danica Direct, Chr. Hansen, Arpajon, France, consisting of Lactococcus lactis ssp. lactis, L. lactis ssp. cremoris, L. lactis ssp. lactis biovar diacetylactis, and Leuconostoc mesenterö̈des spp. cremoris) and a ripening starter $(1.3 \mathrm{~mL}$ of Monilev consisting of Debaryomyces hansenii and Sporendonema casei, and $1.3 \mathrm{~mL}$ of Penbac consisting of Brachybacterium tyrofermentans, Brevibacterium linens, and Penicillium fuscoglaucum, Laboratoire Interprofessionnel de Production (LIP), Aurillac, France) was inoculated on hessian cloths at the stage of moulding. Technological parameters (milk pH, visually assessed rennet clotting time, curd $\mathrm{pH}$, and DM content) were monitored during each cheese-making assay. Overall, 24 cheeses were produced ( 2 grazing systems $\times 2$ treatments of milk $\times 2$ periods $\times 3$ cheese-making dates). The cheeses were ripened for 20 weeks at $10{ }^{\circ} \mathrm{C}$ and $95 \%$ minimum relative humidity and then sampled for analyses.

\subsection{Chemical analyses}

\subsubsection{Milk}

Samples from vat milk used for cheese-making were collected just before the addition of rennet and starter. Immediately, milk pH was determined at $20{ }^{\circ} \mathrm{C}$ using an Ingold needle (Ingold France, Paris, France). Fat, protein and lactose contents were measured (LIAL, Aurillac, France) by infrared spectrometry (Milkoscan 4000, Foss System, Hillerød, Denmark) and, in the same samples, somatic cells (SCC) were automatically counted (Fossomatic 5000, Foss System). The free fatty acid (FFA) content was measured by the copper soap method (Jellema et al., 1991) on fresh samples. Subsamples of milk were stored at $-20{ }^{\circ} \mathrm{C}$ until analysis of lipoprotein 
lipase (LPL) activity and FA composition. The milk LPL activity was measured using an artificial emulsion containing ${ }^{3} \mathrm{H}$ triolein emulsion (Faulconnier, Thévenet, Fléchet, \& Chilliard, 1994). Fatty acid methyl esters in $100 \mathrm{mg}$ samples of lyophilised milk were prepared by direct methylation according to Lerch et al. (2015).

\subsubsection{Cheese}

After ripening, cheeses were cut and divided into triangular pieces of approximately $7 \times 12 \times 3.5 \mathrm{~cm}$ for chemical analyses. Those sub-samples were packed under vacuum in aluminium foil and kept in a plastic bag to preserve antioxidants from oxidation by oxygen or light. Cheese core $\mathrm{pH}$, dry matter (DM) content, and colour were determined as described by Verdier-Metz et al. (2000); fat, calcium, and phosphorus contents were determined as described by Lucas et al. (2006) (Galilait, Theix, France), and total nitrogen (N), water-soluble nitrogen (WSN), and phosphotungstic acid-soluble nitrogen (PTSN) were measured using the methods described by Ardö (1999). The rheological method used was uniaxial compression at a constant displacement rate, as described by Lerch et al. (2015). Resistance to penetration at $10 \%, 30 \%$, and $50 \%$ of the height of the cheese core piece was recorded on five points uniformly distributed over the surface of the test sample. Cheese core and rind samples intended for an FA analysis were stored at $-20^{\circ} \mathrm{C}$. The FA compositions of cheese core and rind were determined in $100 \mathrm{mg}$ samples of lyophilised and ground cheeses as described by Lerch et al. (2015), with one modification compared with milk: methanol/HCl $(95: 5, \mathrm{v} / \mathrm{v})$ was substituted for methanol/boron trifluoride $(95: 5, \mathrm{v} / \mathrm{v})$. Volatile fatty acids (VFA) were solvent extracted and saponified from $15 \mathrm{~g}$ of cheese core, then separated and quantified by gas chromatography coupled to a Flame Ionization Detector (FID) detector (Trace GC Ultra, Thermo Scientific, Gometz le Châtel, France) as described by Bugaud, Buchin, Hauwuy, and Coulon (2001).

\subsection{Microbial analyses}

Milk and cheese samples were stored at $-20^{\circ} \mathrm{C}$ until an analysis was performed. Ten grams of ripened cheese rind or core samples were suspended in $90 \mathrm{~mL}$ phosphate buffer pH 7.5 (Gomri, 1946). Milk and cheese samples were homogenised for 2 min (milk) or $4 \mathrm{~min}$ (cheese) in a Stomacher Laboratory Blender (Interscience, St. Nom la Bretèche, France). This suspension was used for all microbial analyses and appropriate dilutions were plated on the following media (two replicates per sample): heterofermentative facultative Lactobacillus were enumerated on Facultative Heterofermentative (FH) agar medium (Isolini, Grand, \& Glättli, 1990) incubated at $30^{\circ} \mathrm{C}$ for 3 days under anaerobic conditions (Anaerocult A, VWR International, Fontenay-sous-Bois, France), total mesophilic bacteria on Plate Count Agar with Milk (PCAM) medium (IDF, 1990) with milk, Gram-negative bacteria on Plate Count Agar with Inhibitors (PCAI) medium with crystal violet ( $1 \%$ ) and vancomycin $(0.5 \%)$ as inhibitors of the Gram-positive bacteria, lactic acid bacteria on Man Rogosa Sharpe (MRS) (De Man, Rogosa, \& Sharpe, 1960), and dextranproducing Leuconostoc on Mayeux Sandine Elliker (MSE) (Mayeux, Sandine, \& Elliker, 1962) agar medium at $30{ }^{\circ} \mathrm{C}$ for 3 days, yeasts and moulds on Oxytetracycline Glucose Agar (OGA) (Mossel, Visser, \& Merrgerinsk, 1962) at $25{ }^{\circ} \mathrm{C}$ for 3 days, Enterococcus on Slanetz Bartley (SB) agar medium (Slanetz \& Bartley, 1957) at $42{ }^{\circ} \mathrm{C}$ for $48 \mathrm{~h}$, Enterobacteriaceae on Violet Red Bile Glucose (VRBG) agar at $42{ }^{\circ} \mathrm{C}$ for $24 \mathrm{~h}$ (Mossel, Mengerink, \& Scholts, 1962), Gram-positive catalase-positive bacteria on Cheese Ripening Bacteria Medium (CRBM) agar (Denis, Gueguen, Henry, \& Levert, 2001) at $25^{\circ} \mathrm{C}$ for 5 days in the dark then for 5 days under daylight. Ready-to-use media were purchased from Biokar Diagnostics (Biokar Diagnostics, Beauvais, France).

\subsection{Sensory analyses}

The sensory properties of the cheeses were assessed by a panel of 11 expert assessors who performed routine sensory analyses of Cantal-type cheeses (ENILV, Aurillac, France). The selection and training of the panellists were performed as described by Martin et al. (2009). During testing, the panellists gave an intensity score of between 0 and 10 for 35 attributes ( 4 for rind cheese appearance, 3 for core cheese appearance, 2 for touch texture, 3 for mouth texture, 9 for odour, 9 for aroma, and 5 for taste). A score of 0 for a given attribute was defined as not perceptible, while a score of 10 was defined as maximal expression of the attribute in a Cantal-type cheese. During one session, each of the 4 cheeses made on the same day were coded with 3-digit random numbers and presented at $20^{\circ} \mathrm{C}$ to the panellists in a varying order using a monadic sequential method. In all, 6 sessions were necessary to analyse all the cheeses (three sessions on December 2012 for July 2012 cheeses, and three on February 2013 for September 2012 cheeses).

\subsection{Statistical analyses}

Statistical analyses were performed with $\mathrm{R}$ for Windows (version 3.1.0). Milk data were analysed by a multi-way analysis of variance (ANOVA), including variables corresponding to the grazing system used (S), the period of cheese-making (P), and their interactions as fixed factors. Cheese were analysed by ANOVA where variables corresponding to the grazing system used (S), the period $(P)$, the treatment of milk $(T)$, and their interactions were the fixed factors. Sensory data were analysed using a mixed model where the grazing system, the period, the treatment of milk, and their interactions were the fixed factors, and the assessor was the random factor. Significance was declared at $P<0.05$. $P$ values between 0.05 and 0.10 were considered a tendency, while $P>0.10$ was considered not significant. When interactions were not significant, $P$ values were not reported. If significant differences were found by an ANOVA or a mixed-model analysis, the Benjamini-Hochberg method was used to compare differences between multiple groups. A principal component analysis (PCA) was performed on biochemical and microbial data using cheese parameters with significant differences among grazing systems, period, and milk treatment in the ANOVA as active variables and the highest discriminant sensorial attributes as non-active variables.

\section{Results}

\subsection{Milk biochemical characteristics and microbial composition}

Results for milk composition are given in Table 1. For every studied period of cheese-making, the milk fat content and the fat to protein ratio were significantly higher in EXT than in SEMI milks $(P<0.001)$. The SEMI milks had higher $\mathrm{pH}$ and SCC values than EXT milks.

The FFA content of milk was not affected by the grazing system, contrary to the FA profiles. The EXT milks were higher in C18:0

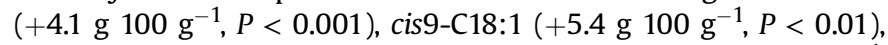
long-chain saturated FA (C20:0 + C22:0 + C24:0; +0.16 g $100 \mathrm{~g}^{-1}$, $P<0.001$ ), cis monounsaturated FA (cis-MUFA; +5.0 g $100 \mathrm{~g}^{-1}$, $P<0.01$ ), and lower in short- and medium-chain FA

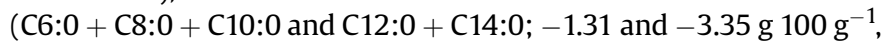
respectively), C16:0 (-3.25 g $\left.100 \mathrm{~g}^{-1}, P<0.01\right)$, trans11-C18:1

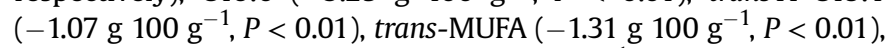

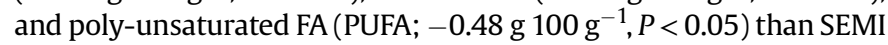
milks. The cis9-C18:1 to $\mathrm{C} 16: 0$ ratio was significantly higher $(P<0.001)$ in EXT than SEMI milks. The fatty acid composition of milks is detailed in Supplementary Table S2. Regarding microbial 
Table 1

Effects of grazing system and period of cheese-making on bulk milk gross composition, fatty acid concentrations and the level of the major microbial groups. ${ }^{\mathrm{a}}$

\begin{tabular}{|c|c|c|c|c|c|c|c|c|}
\hline \multirow[t]{2}{*}{ Item } & \multicolumn{2}{|l|}{ July } & \multicolumn{2}{|c|}{ September } & \multirow[t]{2}{*}{ SEM } & \multicolumn{3}{|c|}{ Effects and significance } \\
\hline & EXT & SEMI & EXT & SEMI & & $\mathrm{S}$ & $\mathrm{P}$ & $\mathrm{S}^{*} \mathrm{P}$ \\
\hline Milk pH & 6.71 & 6.73 & 6.70 & 6.72 & 0.003 & $* *$ & ns & ns \\
\hline Fat content $\left(\mathrm{g} \mathrm{kg}^{-1}\right)$ & 40.1 & 34.4 & 42.1 & 37.3 & 0.910 & $* * *$ & $* *$ & ns \\
\hline Protein content $\left(\mathrm{g} \mathrm{kg}^{-1}\right)$ & $29.1^{\mathrm{b}}$ & $29.8^{\mathrm{b}}$ & $29.9^{\mathrm{b}}$ & $32.9^{\mathrm{a}}$ & 0.466 & $* * *$ & $* * *$ & $* *$ \\
\hline Fat/protein ratio & 1.37 & 1.17 & 1.37 & 1.10 & 0.038 & $* * *$ & ns & ns \\
\hline Lactose content $\left(\mathrm{g} \mathrm{kg}^{-1}\right)$ & $50.3^{\mathrm{a}}$ & $49.3^{\mathrm{a}}$ & $47.8^{\mathrm{b}}$ & $49.2^{\mathrm{ab}}$ & 0.317 & ns & $*$ & $*$ \\
\hline $\operatorname{SCC}\left(10^{3} \mathrm{cfu} \mathrm{mL} \mathrm{m}^{-1}\right)$ & 184 & 262 & 213 & 365 & 23.80 & $* *$ & $*$ & ns \\
\hline FFA (mEq $100 \mathrm{~g}^{-1}$ of fat) & 0.19 & 0.20 & 0.37 & 0.29 & 0.068 & ns & ns & ns \\
\hline LPL activity (nmol min ${ }^{-1} \mathrm{~mL}^{-1}$ ) & 634 & 643 & 641 & 596 & 13.41 & ns & ns & ns \\
\hline \multicolumn{9}{|l|}{$\mathrm{FA}\left(\mathrm{g} 100 \mathrm{~g}^{-1}\right.$ of $\left.\mathrm{FA}\right)$} \\
\hline $\mathrm{C} 4: 0$ & 3.29 & 3.02 & 3.17 & 3.10 & 0.045 & $\bullet$ & ns & ns \\
\hline $\mathrm{C} 6: 0+\mathrm{C} 8: 0+\mathrm{C} 10: 0$ & 4.72 & 6.34 & 4.65 & 5.65 & 0.248 & $* *$ & ns & ns \\
\hline $\mathrm{C} 12: 0+\mathrm{C} 14: 0$ & 10.9 & 14.7 & 11.8 & 14.7 & 0.583 & $* * *$ & ns & ns \\
\hline $\mathrm{C} 16: 0$ & 24.3 & 26.4 & 24.7 & 29.1 & 0.654 & $* *$ & • & ns \\
\hline $\mathrm{C} 18: 0$ & 13.2 & 9.42 & 12.5 & 8.08 & 0.687 & $* * *$ & ns & ns \\
\hline $\mathrm{C} 20: 0+\mathrm{C} 22: 0+\mathrm{C} 24: 0$ & 0.30 & 0.18 & 0.36 & 0.17 & 0.026 & $* * *$ & ns & ns \\
\hline cis9-C18:1 & 24.9 & 19.8 & 23.5 & 17.8 & 0.997 & $* *$ & ns & ns \\
\hline$\Sigma$ other cis-C18:1 & 1.17 & 1.07 & 1.06 & 0.86 & 0.037 & ** & $* *$ & ns \\
\hline trans11-C18:1 & 2.60 & 3.25 & 2.33 & 3.82 & 0.215 & $* *$ & ns & ns \\
\hline$\Sigma$ other trans-C18:1 & 4.06 & 5.04 & 3.77 & 5.41 & 0.265 & $*$ & ns & ns \\
\hline $\mathrm{C} 18: 3 n-3$ & 0.86 & 0.77 & 0.94 & 0.66 & 0.033 & $* * *$ & ns & $* *$ \\
\hline$\Sigma$ CLA & $1.10^{\mathrm{b}}$ & $1.45^{\mathrm{b}}$ & $1.14^{\mathrm{b}}$ & $2.26^{\mathrm{a}}$ & 0.152 & $* * *$ & $*$ & $*$ \\
\hline$\Sigma n-3$ LCPUFA & $1.11^{\mathrm{b}}$ & $0.99^{c}$ & $1.24^{\mathrm{a}}$ & $0.91^{\mathrm{d}}$ & 0.038 & $* * *$ & ns & $* *$ \\
\hline$\Sigma n-6$ LCPUFA & $1.45^{\mathrm{a}}$ & $1.49^{\mathrm{a}}$ & $1.38^{\mathrm{a}}$ & $1.20^{\mathrm{b}}$ & 0.037 & ns & $* *$ & $*$ \\
\hline$\Sigma \mathrm{SFA}^{7}$ & 60.9 & 64.3 & 62.0 & 65.0 & 0.675 & * & ns & ns \\
\hline$\Sigma$ cis-MUFA & 29.0 & 24.2 & 27.7 & 22.5 & 0.939 & ** & ns & ns \\
\hline$\Sigma$ trans-MUFA & 5.06 & 6.00 & 4.79 & 6.47 & 0.268 & $* *$ & ns & ns \\
\hline$\Sigma$ PUFA & 4.16 & 4.51 & 4.33 & 4.94 & 0.117 & $*$ & ns & ns \\
\hline cis9-C18:1/C16:0 & 1.03 & 0.75 & 0.95 & 0.62 & 0.054 & $* * *$ & ns & ns \\
\hline \multicolumn{9}{|l|}{ Presumed microbial group $\left(\log \mathrm{cfu} \mathrm{mL}^{-1}\right.$ ) } \\
\hline Total mesophilic bacteria & 3.22 & 2.96 & 3.46 & 3.11 & 0.070 & * & $\bullet$ & ns \\
\hline Lactic acid bacteria & 3.16 & 2.76 & 3.31 & 3.00 & 0.089 & * & ns & ns \\
\hline Heterofermentative facultative Lactobacillus & 2.83 & 2.32 & 2.95 & 2.60 & 0.097 & * & ns & ns \\
\hline Dextran-producing Leuconostoc & 1.71 & 1.54 & 0.40 & 0.52 & 0.232 & ns & $*$ & ns \\
\hline Gram-positive catalase-positive bacteria & 1.97 & 1.75 & 1.53 & 1.53 & 0.096 & ns & ns & ns \\
\hline Gram-negative bacteria & 2.06 & 1.93 & 1.43 & 1.49 & 0.221 & ns & ns & ns \\
\hline Enterococcus & 0.60 & 0.45 & 0.73 & 0.94 & 0.141 & ns & ns & ns \\
\hline Yeast & 1.42 & 1.30 & 1.38 & 1.02 & 0.138 & ns & ns & ns \\
\hline Mould & 0.77 & 0.48 & 0.91 & 0.92 & 0.092 & ns & ns & ns \\
\hline
\end{tabular}

a Values reported are the mean from triplicate milk samples $(\mathrm{n}=3)$; values with different superscript letters differ at $P<0.05$ by statistical Benjamini-Hochberg test $(\bullet$

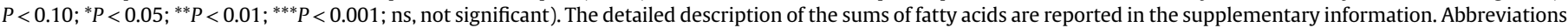

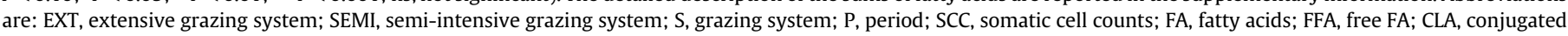
linoleic acid; SFA, saturated FA; PUFA, poly-unsaturated FA; LCPUFA, long-chain PUFA; MUFA, mono-unsaturated FA; SEM, standard error of the mean.

levels, three interrelated microbial groups were affected $(P<0.05)$ by the grazing system regardless of the period studied. The levels of total mesophilic bacteria, lactic acid bacteria, and heterofermentative facultative Lactobacillus were higher in EXT than in SEMI milks $\left(+0.31,+0.36\right.$ and $+0.43 \log \mathrm{cfu} \mathrm{mL}^{-1}$, respectively).

With regard to the period, the milk fat content and SCC increased from July to September. The period had a limited effect on milk FA composition and microbial levels regardless of the grazing system used. The concentration of cis-C18:1 other than oleic acid and the level of dextran-producing Leuconostoc decreased from July to September. In September, milk protein and lactose contents were higher in SEMI milks than in EXT milks (+3.0 and $1.4 \mathrm{~g} \mathrm{~kg}^{-1}$, respectively) while no difference was observed in milks from July. The proportion of $n-3$ FA was higher in EXT than in SEMI milks.

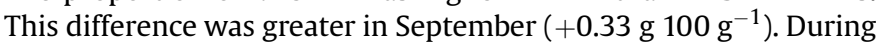
this period, SEMI milks had a higher proportion of conjugated linoleic acids (CLA) but a lower proportion of $n-6$ PUFA than EXT milks.

\subsection{Cheese chemical characteristics and microbial composition}

The rennet clotting time was lower for EXT than SEMI milks ( -2 min 58 s, $P<0.001$; Supplementary Table S3). The acidification of the curd was similar, but the draining was faster in EXT than SEMI cheeses, resulting in a significantly higher DM content in EXT cheeses placed in the cellar $(P<0.001)$. Milk pasteurisation resulted in slightly reduced curd draining, while $R$ and PA cheeses placed in the cellar had similar $\mathrm{pH}$ values. From July to September, the rennet clotting time decreased $(-1 \min 26 \mathrm{~s}, P<0.05)$ and curd draining was slower, even if the cheeses placed in the cellar had similar $\mathrm{pH}$ and dry matter content (DM) values.

For every grazing system and period of cheese-making tested, the fat in DM content was significantly higher in $\mathrm{R}$ than in PA cheeses $(+1.68 \%, \mathrm{P}<0.001$; Table 2$)$. $\mathrm{R}$ cheeses had higher $\mathrm{pH}$ values than $\mathrm{PA}$ cheese $(\mathrm{P}<0.001)$. The maximal deformation strength was lower for $R$ than for PA cheeses $\left(-82 \mathrm{~N} \mathrm{~cm}^{-2}\right.$, $P<0.001)$. The $\mathrm{R}$ cheeses were brighter than the PA cheeses $(P<0.01)$. The milk treatment did not affect the total FA profile of the cheeses, but it significantly reduced the concentration of the major VFA released through lipolysis (Table 3). In cheese rind, the levels of most microbial groups were lower in PA than in R cheeses (Table 4). However, the levels of Gram-positive catalase-positive bacteria tended to be higher in PA cheese rinds. In cheese cores, the levels of Gram-positive catalase-positive bacteria and of Enterobacteriaceae were slightly lower $(P<0.05)$ in PA than in R cheeses. Furthermore, primary (WSN/N) and secondary (PTSN/N) 
Table 2

Effects of grazing system, period and treatment of milk on cheese gross composition, proteolysis, rheology, and colour. ${ }^{\mathrm{a}}$

\begin{tabular}{|c|c|c|c|c|c|c|c|c|c|c|c|c|c|c|}
\hline \multirow[t]{3}{*}{ Parameters } & \multicolumn{4}{|l|}{ Raw } & \multicolumn{4}{|c|}{ Pasteurised } & \multirow[t]{3}{*}{ SEM } & \multicolumn{5}{|c|}{ Effects and significance } \\
\hline & \multicolumn{2}{|l|}{ July } & \multicolumn{2}{|c|}{ September } & \multicolumn{2}{|l|}{ July } & \multicolumn{2}{|c|}{ September } & & \multirow[t]{2}{*}{$\mathrm{S}$} & \multirow[t]{2}{*}{$\mathrm{T}$} & \multirow[t]{2}{*}{$\mathrm{S}^{*} \mathrm{~T}$} & \multirow[t]{2}{*}{$\mathrm{P}$} & \multirow[t]{2}{*}{$\mathrm{P}^{*} \mathrm{~T}$} \\
\hline & EXT & SEMI & EXT & SEMI & EXT & SEMI & EXT & SEMI & & & & & & \\
\hline DM (\%) & $63.6^{\mathrm{abc}}$ & $63.6^{\mathrm{abc}}$ & $65.0^{\mathrm{a}}$ & $62.8^{c}$ & $63.6^{\mathrm{abc}}$ & $64.4^{\mathrm{ab}}$ & $63.4^{\mathrm{bc}}$ & $63.8^{\mathrm{abc}}$ & 0.199 & ns & ns & $*$ & ns & ns \\
\hline Fat (\%) & $35.4^{\mathrm{ab}}$ & $32.4^{\mathrm{de}}$ & $36.1^{\mathrm{a}}$ & $31.8^{\mathrm{e}}$ & $34.3^{b c}$ & $32.2^{\text {de }}$ & $33.5^{\mathrm{cd}}$ & $31.5^{\mathrm{e}}$ & 0.360 & $* * *$ & $* *$ & $*$ & ns & ns \\
\hline Fat in DM (\%) & 55.7 & 51.0 & 55.5 & 50.7 & 54.0 & 50.0 & 52.8 & 49.4 & 0.506 & $* * *$ & $* * *$ & ns & ns & ns \\
\hline $\mathrm{pH}$ & 5.56 & 5.60 & 5.67 & 5.57 & 5.36 & 5.47 & 5.44 & 5.42 & 0.025 & ns & $* * *$ & ns & ns & ns \\
\hline Calcium (\%) & 0.66 & 0.76 & 0.67 & 0.76 & 0.69 & 0.74 & 0.72 & 0.73 & 0.011 & $* *$ & ns & $\bullet$ & ns & ns \\
\hline Phosphorus (\%) & 0.46 & 0.53 & 0.47 & 0.53 & 0.47 & 0.53 & 0.48 & 0.51 & 0.008 & $* * *$ & ns & ns & ns & ns \\
\hline \multicolumn{15}{|l|}{ Proteolysis } \\
\hline WSN/N (\%) & $26.6^{\mathrm{a}}$ & $23.0^{\mathrm{b}}$ & $21.3^{\mathrm{bc}}$ & $19.8^{c}$ & $20.1^{\mathrm{c}}$ & $20.8^{\mathrm{bc}}$ & $20.9^{\mathrm{bc}}$ & $21.0^{\mathrm{bc}}$ & 0.510 & ns & $* *$ & $*$ & $*$ & $* *$ \\
\hline PTSN/WSN (\%) & 17.7 & 15.7 & 16.2 & 15.1 & 12.6 & 11.2 & 13.4 & 12.4 & 0.528 & $\bullet$ & $* * *$ & ns & ns & ns \\
\hline PTSN/N (\%) & $4.70^{\mathrm{a}}$ & $3.60^{\mathrm{b}}$ & $3.42^{\mathrm{bc}}$ & $2.99^{\mathrm{bcd}}$ & $2.55^{\mathrm{de}}$ & $2.33^{\mathrm{e}}$ & $2.79^{\text {cde }}$ & $2.61^{\mathrm{de}}$ & 0.163 & $* *$ & $* * *$ & $\bullet$ & $*$ & $* *$ \\
\hline \multicolumn{15}{|l|}{ Rheology } \\
\hline Strength to $10 \%$ of deformation $\left(\mathrm{N} \mathrm{cm}^{-2}\right)$ & 1.41 & 1.48 & 1.28 & 1.53 & 1.71 & 1.92 & 1.70 & 1.97 & 0.071 & ns & $* *$ & ns & ns & ns \\
\hline Strength to $30 \%$ of deformation $\left(\mathrm{N} \mathrm{cm}^{-2}\right)$ & 3.59 & 4.27 & 3.40 & 3.83 & 5.38 & 5.88 & 4.88 & 5.60 & 0.218 & $*$ & $* * *$ & ns & ns & ns \\
\hline Strength to $50 \%$ of deformation $\left(\mathrm{N} \mathrm{cm}^{-2}\right)$ & 5.36 & 6.43 & 5.11 & 6.17 & 8.14 & 9.46 & 7.56 & 8.71 & 0.353 & $* *$ & $* * *$ & ns & ns & ns \\
\hline Strength to max deformation $\left(\mathrm{N} \mathrm{cm}^{-2}\right)$ & 206 & 250 & 209 & 259 & 290 & 353 & 276 & 332 & 11.50 & $* * *$ & $* * *$ & ns & ns & ns \\
\hline \multicolumn{15}{|l|}{ Colour (cheese core) } \\
\hline$L^{*}$ (black-white) & 76.9 & 75.3 & 78.4 & 78.7 & 75.9 & 74.6 & 78.1 & 77.6 & 0.315 & $* *$ & $* *$ & ns & $* * *$ & ns \\
\hline$a^{*}($ green-red $)$ & 1.76 & 2.18 & 1.90 & 2.08 & 1.90 & 1.98 & 1.91 & 2.09 & 0.039 & $* *$ & ns & ns & ns & ns \\
\hline$b^{*}($ blue-yellow) & $20.0^{\mathrm{bc}}$ & $21.1^{\mathrm{a}}$ & $19.6^{c}$ & $20.4^{\mathrm{ab}}$ & $20.5^{\mathrm{ab}}$ & $21.0^{\mathrm{a}}$ & $21.0^{\mathrm{a}}$ & $20.9^{\mathrm{a}}$ & 0.124 & $* *$ & $* *$ & $*$ & ns & $\bullet$ \\
\hline
\end{tabular}

a Values reported are the mean from triplicate cheese samples $(\mathrm{n}=3$ ); values with different superscript letters differ at $P<0.05$ by statistical Benjamini-Hochberg test $(\bullet$

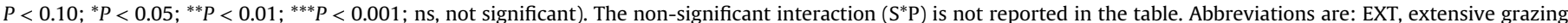

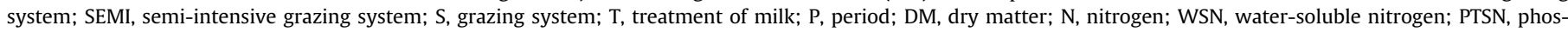
photungstic acid soluble nitrogen; SEM, standard error of the means.

Table 3

Effects of grazing system, period of cheese-making and treatment of milk on concentrations of fatty acids and volatile fatty acids in cheeses. ${ }^{a}$

\begin{tabular}{|c|c|c|c|c|c|c|c|c|c|c|c|c|c|}
\hline \multirow[t]{3}{*}{ Fatty acids (g $100 \mathrm{~g}^{-1}$ of FA) } & \multicolumn{4}{|l|}{ Raw } & \multicolumn{4}{|c|}{ Pasteurised } & \multirow[t]{3}{*}{ SEM } & \multirow{2}{*}{\multicolumn{4}{|c|}{ Effects and significance }} \\
\hline & \multicolumn{2}{|l|}{ July } & \multicolumn{2}{|c|}{ September } & \multicolumn{2}{|l|}{ July } & \multicolumn{2}{|c|}{ September } & & & & & \\
\hline & EXT & SEMI & EXT & SEMI & EXT & SEMI & EXT & SEMI & & $\mathrm{S}$ & $\mathrm{T}$ & $P$ & $\mathrm{~S}^{*} \mathrm{P}$ \\
\hline $\mathrm{C} 4: 0$ & 3.77 & 3.42 & 3.35 & 3.56 & 3.68 & 3.60 & 3.32 & 3.41 & 0.051 & ns & ns & * & $\bullet$ \\
\hline $\mathrm{C} 6: 0+\mathrm{C} 8: 0+\mathrm{C} 10: 0$ & 4.91 & 6.35 & 4.61 & 6.01 & 4.85 & 6.54 & 4.68 & 5.78 & 0.178 & $* * *$ & ns & • & ns \\
\hline $\mathrm{C} 12: 0+\mathrm{C} 14: 0$ & 10.9 & 14.7 & 11.7 & 14.9 & 11.0 & 14.5 & 11.8 & 14.8 & 0.393 & $* * *$ & ns & ns & ns \\
\hline C16:0 & $24.0^{c}$ & $26.2^{\mathrm{b}}$ & $24.5^{\mathrm{bc}}$ & $28.7^{\mathrm{a}}$ & $23.9^{c}$ & $25.8^{\mathrm{bc}}$ & $24.5^{\mathrm{bc}}$ & $28.9^{\mathrm{a}}$ & 0.444 & $* * *$ & ns & $* *$ & $*$ \\
\hline C18:0 & 13.2 & 9.45 & 12.5 & 7.98 & 13.0 & 9.38 & 12.4 & 7.99 & 0.468 & $* * *$ & ns & $*$ & $\mathrm{~ns}$ \\
\hline $\mathrm{C} 20: 0+\mathrm{C} 22: 0+\mathrm{C} 24: 0$ & $0.31^{\mathrm{b}}$ & $0.20^{c}$ & $0.38^{\mathrm{a}}$ & $0.16^{\mathrm{c}}$ & $0.31^{\mathrm{b}}$ & $0.19^{c}$ & $0.38^{\mathrm{ab}}$ & $0.17^{\mathrm{c}}$ & 0.019 & $* * *$ & ns & ns & $*$ \\
\hline cis9-C18:1 & 24.6 & 19.5 & 23.2 & 17.4 & 25.0 & 19.8 & 23.2 & 17.6 & 0.680 & $* * *$ & ns & $*$ & ns \\
\hline$\Sigma$ cis-C18:1 isomers & $1.50^{\mathrm{a}}$ & $1.40^{\mathrm{ab}}$ & $1.40^{\mathrm{ab}}$ & $1.11^{\mathrm{c}}$ & $1.48^{\mathrm{ab}}$ & $1.39^{\mathrm{b}}$ & $1.41^{\mathrm{ab}}$ & $1.13^{\mathrm{c}}$ & 0.031 & $* * *$ & ns & $* * *$ & $* *$ \\
\hline trans11-C18:1 & 2.61 & 3.34 & 2.37 & 3.82 & 2.62 & 3.33 & 2.45 & 3.85 & 0.149 & $* * *$ & ns & ns & ns \\
\hline$\Sigma$ trans-C18:1 isomers & 3.64 & 4.61 & 3.44 & 5.09 & 3.57 & 4.63 & 3.52 & 5.14 & 0.180 & $* * *$ & ns & ns & ns \\
\hline $\mathrm{C} 18: 3 n-3$ & 0.88 & 0.78 & 0.95 & 0.65 & 0.89 & 0.80 & 0.94 & 0.66 & 0.024 & $* * *$ & ns & $\bullet$ & $* * *$ \\
\hline$\Sigma$ CLA & $1.06^{\mathrm{b}}$ & $1.44^{\mathrm{b}}$ & $1.13^{\mathrm{b}}$ & $2.15^{\mathrm{a}}$ & $1.06^{\mathrm{b}}$ & $1.45^{\mathrm{b}}$ & $1.19^{\mathrm{b}}$ & $2.21^{\mathrm{a}}$ & 0.099 & $* * *$ & ns & $* * *$ & $* *$ \\
\hline$\sum n-3$ LCPUFA & $1.15^{\mathrm{b}}$ & $1.03^{\mathrm{c}}$ & $1.26^{\mathrm{a}}$ & $0.89^{\mathrm{d}}$ & $1.15^{\mathrm{b}}$ & $1.05^{\mathrm{c}}$ & $1.26^{\mathrm{a}}$ & $0.90^{\mathrm{d}}$ & 0.029 & $* * *$ & ns & ns & $* * *$ \\
\hline$\Sigma n-6$ LCPUFA & $1.47^{\mathrm{a}}$ & $1.52^{\mathrm{a}}$ & $1.43^{\mathrm{a}}$ & $1.16^{\mathrm{b}}$ & $1.48^{\mathrm{a}}$ & $1.53^{\mathrm{a}}$ & $1.44^{\mathrm{a}}$ & $1.20^{\mathrm{b}}$ & 0.031 & $* *$ & ns & $* * *$ & $* * *$ \\
\hline$\Sigma$ SFA & 61.3 & 64.6 & 62.1 & 65.6 & 60.9 & 64.2 & 62.0 & 65.3 & 0.454 & $* * *$ & ns & ns & ns \\
\hline$\Sigma$ cis-MUFA & 28.7 & 23.9 & 27.5 & 22.1 & 29.1 & 24.2 & 27.5 & 22.3 & 0.637 & $* * *$ & ns & $*$ & ns \\
\hline$\Sigma$ trans-MUFA & 4.95 & 5.89 & 4.81 & 6.40 & 4.88 & 5.90 & 4.88 & 6.44 & 0.179 & $* * *$ & ns & ns & ns \\
\hline$\Sigma$ PUFA & 4.19 & 4.62 & 4.42 & 4.77 & 4.20 & 4.67 & 4.49 & 4.88 & 0.072 & $* *$ & ns & • & ns \\
\hline cis9-C18:1/C16:0 & 1.02 & 0.75 & 0.95 & 0.61 & 1.04 & 0.77 & 0.95 & 0.61 & 0.038 & $* * *$ & ns & $* *$ & ns \\
\hline \multicolumn{14}{|l|}{ Volatile fatty acids } \\
\hline Acetic acid & 61.1 & 72.1 & 56.6 & 64.5 & 46.7 & 43.1 & 33.5 & 43.9 & 3.393 & ns & $* *$ & ns & ns \\
\hline Propionic acid & 0.35 & 0.70 & 1.58 & 0.32 & 0.14 & 0.10 & 0.09 & 0.08 & 0.137 & ns & $*$ & ns & - \\
\hline Isobutyric acid & $0.16^{\mathrm{abc}}$ & $0.23^{\mathrm{a}}$ & $0.19^{\mathrm{ab}}$ & $0.08^{\mathrm{de}}$ & $0.12^{\text {bcd }}$ & $0.10^{\text {cde }}$ & $0.10^{\text {cde }}$ & $0.05^{\mathrm{e}}$ & 0.013 & $\bullet$ & **** & $* *$ & $* *$ \\
\hline Butyric acid & $15.7^{\mathrm{b}}$ & $13.8^{\mathrm{bc}}$ & $20.5^{\mathrm{a}}$ & $10.3^{\text {cde }}$ & $9.88^{\text {def }}$ & $7.50^{\text {ef }}$ & $11.4^{\mathrm{cd}}$ & $6.44^{\mathrm{f}}$ & 0.963 & $* * *$ & $* * *$ & ns & $* *$ \\
\hline Isovaleric acid & $0.38^{\mathrm{abc}}$ & $0.42^{\mathrm{abc}}$ & $0.45^{\mathrm{ab}}$ & $0.21^{\mathrm{bc}}$ & $0.32^{\mathrm{abc}}$ & $0.50^{\mathrm{a}}$ & $0.36^{\mathrm{abc}}$ & $0.20^{c}$ & 0.031 & ns & ns & $\bullet$ & $*$ \\
\hline Isocaproic acid & 0.06 & 0.06 & 0.04 & 0.05 & 0.05 & 0.06 & 0.05 & 0.05 & 0.004 & ns & ns & ns & ns \\
\hline Caproic acid & $6.04^{\mathrm{b}}$ & $6.07^{\mathrm{b}}$ & $7.76^{\mathrm{a}}$ & $5.06^{\mathrm{bc}}$ & $3.94^{\mathrm{cd}}$ & $3.66^{\mathrm{cd}}$ & $4.74^{\mathrm{bc}}$ & $2.62^{d}$ & 0.348 & $* *$ & $* * *$ & ns & $* *$ \\
\hline
\end{tabular}

a Values reported are the mean from triplicate cheese samples $(\mathrm{n}=3$ ); values with different superscript letters differ at $P<0.05$ by statistical Benjamini-Hochberg test $(\bullet$

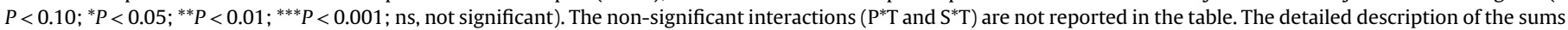

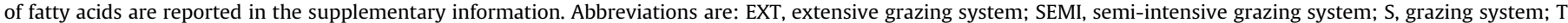

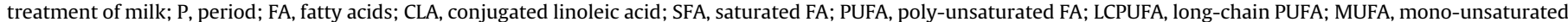
FA; SEM, standard error of the means. 
Table 4

Effects of grazing system, period of cheese-making and treatment of milk on the level of the major microbial groups in rind and core of cheeses.

\begin{tabular}{|c|c|c|c|c|c|c|c|c|c|c|c|c|c|}
\hline \multirow[t]{3}{*}{ Presumed microbial group (log cfu g ${ }^{-1}$ ) } & \multicolumn{4}{|l|}{ Raw } & \multicolumn{4}{|c|}{ Pasteurised } & \multirow[t]{3}{*}{ SEM } & \multirow{2}{*}{\multicolumn{4}{|c|}{ Effects and significance }} \\
\hline & \multicolumn{2}{|l|}{ July } & \multicolumn{2}{|c|}{ September } & \multicolumn{2}{|l|}{ July } & \multicolumn{2}{|c|}{ September } & & & & & \\
\hline & EXT & SEMI & EXT & SEMI & EXT & SEMI & EXT & SEMI & & S & $\mathrm{T}$ & $\mathrm{P}$ & $\mathrm{P}^{*} \mathrm{~T}$ \\
\hline \multicolumn{14}{|l|}{ Cheese rind } \\
\hline Total mesophilic bacteria & 9.20 & 9.42 & 9.00 & 9.11 & 9.47 & 9.17 & 9.22 & 9.16 & 0.049 & ns & ns & $\bullet$ & ns \\
\hline Lactic acid bacteria & 7.27 & 6.93 & 7.32 & 7.23 & 6.43 & 6.08 & 6.31 & 6.36 & 0.108 & • & $* * *$ & ns & ns \\
\hline Heterofermentative facultative Lactobacillus & $7.24^{\mathrm{a}}$ & $6.82^{\mathrm{a}}$ & $7.34^{\mathrm{a}}$ & $7.04^{\mathrm{a}}$ & $4.92^{\mathrm{C}}$ & $4.52^{\mathrm{C}}$ & $5.98^{\mathrm{b}}$ & $5.99^{\mathrm{b}}$ & 0.223 & ns & $* * *$ & $* *$ & * \\
\hline Gram-positive catalase-positive bacteria & 9.71 & 9.98 & 9.70 & 9.47 & 10.0 & 10.1 & 9.94 & 9.86 & 0.072 & ns & $\bullet$ & ns & ns \\
\hline Enterococcus & 5.45 & 5.20 & 5.33 & 5.07 & nd & nd & 1.94 & 1.70 & 0.417 & ns & $* * *$ & ns & • \\
\hline Enterobacteriaceae & 2.74 & 2.35 & 2.75 & 2.45 & nd & nd & 2.15 & nd & 0.190 & $\bullet$ & $* * *$ & ns & ns \\
\hline Yeast & 6.81 & 6.49 & 6.26 & 5.95 & 4.73 & 4.18 & 5.38 & 3.74 & 0.253 & * & $* * *$ & ns & ns \\
\hline Mould & 6.50 & 6.73 & 6.66 & 6.71 & 6.05 & 6.13 & 5.93 & 6.16 & 0.091 & ns & $* *$ & ns & ns \\
\hline \multicolumn{14}{|l|}{ Cheese core } \\
\hline Total mesophilic bacteria & 7.14 & 6.93 & 7.15 & 7.27 & 7.12 & 6.75 & 7.15 & 7.08 & 0.071 & ns & ns & ns & ns \\
\hline Lactic acid bacteria & 6.15 & 6.91 & 6.64 & 6.93 & 6.99 & 6.78 & 6.65 & 6.68 & 0.160 & ns & ns & ns & ns \\
\hline Heterofermentative facultative Lactobacillus & 7.31 & 6.95 & 7.50 & 7.41 & 7.20 & 6.95 & 7.07 & 6.95 & 0.113 & ns & ns & ns & ns \\
\hline Dextran-producing Leuconostoc & 4.30 & 2.99 & nd & nd & 1.67 & 1.67 & nd & nd & 0.336 & ns & ns & $*$ & ns \\
\hline Gram-positive catalase-positive bacteria & 5.52 & 5.26 & 5.84 & 5.78 & 4.68 & 4.69 & 5.19 & 5.02 & 0.156 & ns & * & ns & ns \\
\hline Enterococcus & $5.95^{\mathrm{a}}$ & $5.76^{\mathrm{a}}$ & $5.45^{\mathrm{a}}$ & $4.94^{\mathrm{a}}$ & nd & nd & $2.76^{\mathrm{b}}$ & $2.08^{\mathrm{bc}}$ & 0.433 & ns & $* * *$ & ns & $* *$ \\
\hline Enterobacteriaceae & 2.67 & 2.30 & 1.63 & 1.43 & nd & nd & 1.72 & nd & 0.178 & ns & * & ns & • \\
\hline Yeast & 3.54 & 2.67 & 4.06 & 3.83 & nd & nd & 3.55 & 3.35 & 0.377 & ns & $\bullet$ & $*$ & ns \\
\hline Mould & 1.08 & 2.19 & nd & nd & 1.41 & nd & nd & 1.49 & 0.125 & ns & ns & ns & ns \\
\hline
\end{tabular}

a Values reported are the mean from triplicate cheese samples $(\mathrm{n}=3)$; values with different superscript letters differ at $P<0.05$ by statistical Benjamini-Hochberg test $(\bullet$

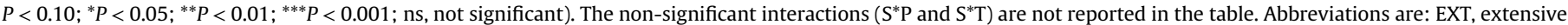

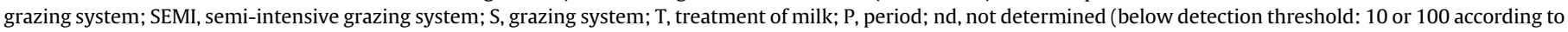
media); SEM, standard error of the means.

proteolysis was most advanced in R cheeses made in July, while no difference was observed among PA cheeses (Table 2). In September, the levels of heterofermentative facultative Lactobacillus on the cheese rind and of Enterococcus on the cheese core were higher in PA cheeses, while no difference was observed among R cheeses (Table 4).

For every milk treatment and period of cheese-making studied, the fat in DM content was significantly higher in EXT than in SEMI cheeses $(+4.23 \%, P<0.001$; Table 2$)$, and the opposite was observed for cheese calcium and phosphorus contents. The maximal deformation strength was higher for SEMI than for EXT cheeses $\left(+53.3 \mathrm{~N} \mathrm{~cm}^{-2}, P<0.001\right)$. The latter were also brighter and less red than SEMI cheeses $(P<0.01)$. The FA profiles of EXT and SEMI cheeses were very different, as they were for their respective milks (Table 3). The EXT cheese cores were higher in C18:0 $(+4.1 \mathrm{~g}$ $\left.100 \mathrm{~g}^{-1}, P<0.001\right)$, cis9-C18:1 (+5.4 g $\left.100 \mathrm{~g}^{-1}, P<0.001\right)$, and cis-

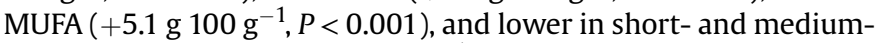

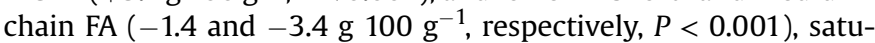
rated FA (SFA; $\left.-3.4 \mathrm{~g} 100 \mathrm{~g}^{-1}, P<0.001\right)$, trans11-C18:1 $(-1.1 \mathrm{~g}$

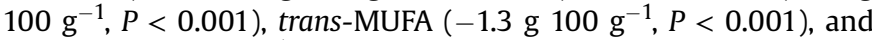

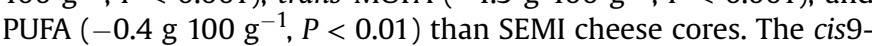
C18:1 to C16:0 ratio was significantly higher for EXT than for SEMI cheese cores $(P<0.001)$. The fatty acid composition of cheese cores is detailed in Supplementary Table S4. Similar results were obtained for cheese rinds (Supplementary Table S5). The levels of microbial groups in cheeses were weakly impacted by the grazing system used, except for yeasts (Table 4). Their levels were higher in EXT than in SEMI cheese rinds $\left(+0.71 \log \mathrm{cfu}^{-1}\right)$.

The interaction between the effects of the grazing system and milk pasteurisation $\left(\mathrm{S}^{*} \mathrm{~T}\right)$ was significant for some cheese characteristics: the DM content of SEMI cheeses was higher in PA than in $\mathrm{R}$ cheeses, contrary to the DM content of EXT cheeses (Table 2). Fat content in EXT cheeses was higher in R than in PA cheeses, while no difference was observed in SEMI cheeses. The indicator of primary proteolysis (WSN/N) in R cheeses was higher in EXT cheeses than in SEMI cheeses, while it was equivalent in all PA cheeses regardless of the grazing system used. The colour of $\mathrm{R}$ cheeses was less yellow than that of PA cheeses, in particular for EXT cheeses.
Concerning the period of cheese-making, the gross composition of ripened cheeses made in July and September was similar regardless of the grazing system used and any milk treatment (Table 2). Cheeses made in July were less white than those made in September $(P<0.001)$. The concentrations of $\mathrm{C} 18: 0(+1.1 \mathrm{~g}$

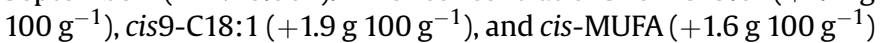
were slightly higher in July than in September cheese cores $(P<0.05$; Table 3$)$. The level of dextran-producing Leuconostoc was higher in the cores of cheeses made in July, while the opposite effect was observed for yeasts $(P<0.05$; Table 4$)$. In September, the concentrations of C16:0 and CLA were higher in SEMI than in EXT cheese cores (Table 3). During this period, SEMI cheese cores had lower concentrations of long-chain saturated FA, $n-3$ FA, and $n-6$ FA. Moreover, in the volatile fraction, the concentrations of butyric and caproic acids were higher for EXT than for SEMI cheeses, especially in September.

\subsection{Cheese sensory properties}

Cantal-type cheese sensory characteristics are presented in Table 5. For every grazing system and period of cheese-making studied, most sensory attributes were modified by the milk treatment used. In $\mathrm{R}$ cheese rinds, the spot salience $(-3.0)$ and quantity $(-3.3)$ were scored lower than for PA cheeses. The R cheese cores were less yellow $(-0.4, P<0.001)$ and less veined $(-0.6, P<0.01)$ than PA cheese cores. The $\mathrm{R}$ cheeses were considered by the panellists to be less firm to the touch $(-0.9, P<0.001)$ and more granular in the mouth $(+0.5, P<0.001)$ than PA cheeses. The $\mathrm{R}$ cheeses were characterised by a more intense taste $(+0.9$, $P<0.001)$ with a more pronounced pungent taste $(+0.7, P<0.001)$ than PA cheeses. The R cheeses also developed more intense odours $(+0.9, P<0.01)$ characterised by the attributes "fermented cream" and "yeast", while the PA cheeses were characterised by the attributes "fresh cream" and "butter". The $\mathrm{R}$ cheeses also developed more persistent aromas $(+0.8, P<0.001)$ described as more "rancid" and "stable" and less "butter". The colour of $\mathrm{R}$ cheeses spots was slightly more ochre in September, contrary to those of PA cheeses, which were more ochre in July. Cheeses made in July from 
Table 5

Effects of grazing system, period of cheese-making and treatment of milk on the Cantal-type cheese sensory characteristics. ${ }^{\mathrm{a}}$

\begin{tabular}{|c|c|c|c|c|c|c|c|c|c|c|c|c|c|c|}
\hline \multirow[t]{3}{*}{ Item } & \multicolumn{4}{|l|}{ Raw } & \multicolumn{4}{|c|}{ Pasteurised } & \multirow[t]{3}{*}{ SEM } & \multirow{2}{*}{\multicolumn{5}{|c|}{ Effects and significance }} \\
\hline & \multicolumn{2}{|l|}{ July } & \multicolumn{2}{|c|}{ September } & \multicolumn{2}{|l|}{ July } & \multicolumn{2}{|c|}{ September } & & & & & & \\
\hline & EXT & SEMI & EXT & SEMI & EXT & SEMI & EXT & SEMI & & $\mathrm{S}$ & $\mathrm{T}$ & $\mathrm{S} * \mathrm{~T}$ & $\mathrm{P}$ & $\mathrm{P}^{*} \mathrm{~T}$ \\
\hline \multicolumn{15}{|l|}{ Appearance of cheese rind } \\
\hline White-grey colour & $4.5^{\mathrm{bc}}$ & $4.6^{\mathrm{bc}}$ & $4.2^{\mathrm{c}}$ & $4.1^{\mathrm{c}}$ & $6.1^{\mathrm{a}}$ & $5.6^{\mathrm{a}}$ & $6.2^{\mathrm{a}}$ & $5.3^{\mathrm{ab}}$ & 0.115 & $* *$ & $* * *$ & * & ns & ns \\
\hline Spot colour (white-ochre) & $5.7^{\mathrm{c}}$ & $6.0^{c}$ & $6.0^{c}$ & $6.4^{\mathrm{bc}}$ & $7.6^{\mathrm{a}}$ & $7.5^{\mathrm{ab}}$ & $7.2^{\mathrm{ab}}$ & $7.4^{\mathrm{ab}}$ & 0.070 & ns & $* * *$ & ns & ns & $*$ \\
\hline Spot salience & 4.4 & 4.4 & 4.0 & 4.4 & 7.1 & 7.3 & 7.5 & 7.0 & 0.063 & ns & $* * *$ & ns & ns & ns \\
\hline Spot quantity & 4.1 & 4.3 & 3.0 & 4.2 & 7.0 & 7.7 & 6.9 & 7.3 & 0.074 & $*$ & $* * *$ & ns & ns & ns \\
\hline \multicolumn{15}{|l|}{ Appearance of cheese core } \\
\hline White-yellow colour & 5.6 & 6.3 & 5.4 & 5.8 & 6.4 & 6.7 & 5.6 & 6.0 & 0.061 & $*$ & $* * *$ & ns & $* * *$ & • \\
\hline Core veined & 3.6 & 3.8 & 3.8 & 4.3 & 4.4 & 4.8 & 4.2 & 4.6 & 0.117 & ns & $* *$ & ns & ns & ns \\
\hline Core marbled & 4.6 & 5.2 & 3.5 & 4.0 & 4.6 & 4.5 & 3.4 & 3.7 & 0.110 & ns & ns & ns & $* * *$ & ns \\
\hline \multicolumn{15}{|l|}{ Odour } \\
\hline Intense & 6.3 & 5.7 & 5.5 & 5.6 & 5.2 & 5.0 & 4.9 & 4.6 & 0.093 & ns & $* *$ & ns & • & ns \\
\hline Fresh cream & 0.2 & 0.4 & 0.3 & 0.0 & 0.8 & 0.7 & 0.9 & 0.7 & 0.073 & ns & $* *$ & ns & ns & ns \\
\hline Fermented cream & 1.8 & 1.5 & 0.9 & 1.1 & 0.9 & 0.9 & 0.6 & 0.4 & 0.043 & ns & $* *$ & ns & $\bullet$ & ns \\
\hline Butter & 0.2 & 0.2 & 0.1 & 0.2 & 0.6 & 0.5 & 0.5 & 0.3 & 0.034 & ns & $* * *$ & ns & ns & ns \\
\hline Egg & 0.2 & 0.1 & 0.3 & 0.3 & 0.1 & 0.1 & 0.1 & 0.1 & 0.027 & ns & ns & ns & ns & ns \\
\hline Swiss type cheese & $0.4^{\mathrm{ab}}$ & $0.3^{\mathrm{b}}$ & $0.6^{\mathrm{ab}}$ & $0.4^{\mathrm{ab}}$ & $0.7^{\mathrm{ab}}$ & $0.8^{\mathrm{a}}$ & $0.4^{\mathrm{ab}}$ & $0.5^{\mathrm{ab}}$ & 0.067 & ns & $\bullet$ & ns & $*$ & $*$ \\
\hline Yeast & 1.3 & 0.8 & 0.6 & 0.6 & 0.5 & 0.7 & 0.5 & 0.4 & 0.042 & ns & $* *$ & ns & ns & ns \\
\hline Vanilla & 0.2 & 0.1 & 0.1 & 0.1 & 0.2 & 0.2 & 0.2 & 0.2 & 0.025 & ns & ns & ns & ns & ns \\
\hline Plastic & 0.2 & 0.1 & 0.2 & 0.2 & 0.1 & 0.1 & 0.1 & 0.0 & 0.008 & ns & ns & ns & ns & ns \\
\hline \multicolumn{15}{|l|}{ Texture } \\
\hline Firm & 4.6 & 4.9 & 4.9 & 5.0 & 5.8 & 5.7 & 5.5 & 5.9 & 0.066 & ns & $* * *$ & ns & ns & ns \\
\hline Elastic & $3.8^{\mathrm{d}}$ & $4.5^{c}$ & $4.9^{\mathrm{abc}}$ & $5.5^{\mathrm{a}}$ & $4.8^{\mathrm{bc}}$ & $5.3^{\mathrm{ab}}$ & $5.3^{\mathrm{ab}}$ & $5.0^{\mathrm{abc}}$ & 0.103 & ns & $* * *$ & $*$ & ns & $* * *$ \\
\hline Creamy & 5.2 & 5.0 & 5.5 & 5.1 & 4.8 & 4.3 & 5.0 & 4.6 & 0.068 & $*$ & $\bullet$ & ns & ns & ns \\
\hline Adhesive & 2.1 & 1.9 & 2.6 & 1.8 & 2.6 & 1.6 & 2.7 & 2.3 & 0.097 & $* *$ & ns & ns & ns & ns \\
\hline Granular & 1.7 & 1.4 & 1.1 & 1.0 & 1.0 & 1.0 & 0.5 & 0.9 & 0.054 & ns & $* * *$ & ns & $\bullet$ & ns \\
\hline \multicolumn{15}{|l|}{ Taste } \\
\hline Intense & 6.7 & 6.4 & 6.4 & 6.1 & 5.6 & 5.7 & 5.4 & 5.4 & 0.080 & ns & $* * *$ & ns & $*$ & ns \\
\hline Salty & 5.4 & 5.5 & 5.5 & 5.4 & 5.4 & 5.3 & 5.4 & 5.2 & 0.031 & $*$ & ns & ns & ns & ns \\
\hline Sour & 1.7 & 1.8 & 1.8 & 1.7 & 1.6 & 1.2 & 2.0 & 1.4 & 0.088 & $*$ & ns & $\bullet$ & ns & ns \\
\hline Bitter & 2.2 & 2.2 & 2.5 & 2.0 & 2.0 & 1.8 & 1.9 & 1.7 & 0.090 & ns & ns & ns & ns & ns \\
\hline Pungent & 2.3 & 2.2 & 2.2 & 1.5 & 1.5 & 1.2 & 1.4 & 1.2 & 0.078 & ns & $* * *$ & ns & ns & ns \\
\hline \multicolumn{15}{|l|}{ Aroma } \\
\hline Fresh cream & 0.1 & 0.0 & 0.1 & 0.1 & 0.2 & 0.1 & 0.3 & 0.1 & 0.024 & ns & ns & ns & ns & ns \\
\hline Fermented cream & $1.1^{\mathrm{a}}$ & $0.6^{\mathrm{b}}$ & $0.5^{\mathrm{b}}$ & $0.4^{\mathrm{b}}$ & $0.4^{\mathrm{b}}$ & $0.6^{\mathrm{b}}$ & $0.2^{\mathrm{b}}$ & $0.4^{\mathrm{b}}$ & 0.041 & ns & $* * *$ & $* *$ & ns & ns \\
\hline Butter & 0.0 & 0.1 & 0.1 & 0.1 & 0.2 & 0.3 & 0.2 & 0.1 & 0.017 & ns & $* *$ & ns & $*$ & - \\
\hline Swiss type cheese & 0.2 & 0.2 & 0.2 & 0.1 & 0.3 & 0.4 & 0.2 & 0.3 & 0.048 & ns & ns & ns & $\bullet$ & ns \\
\hline Mushroom & 0.2 & 0.0 & 0.1 & 0.1 & 0.2 & 0.1 & 0.1 & 0.0 & 0.012 & ns & ns & ns & ns & ns \\
\hline Stable & 0.8 & 0.5 & 0.4 & 0.3 & 0.2 & 0.3 & 0.1 & 0.1 & 0.023 & ns & $* * *$ & ns & ns & ns \\
\hline Rancid & 0.6 & 0.6 & 0.5 & 0.4 & 0.2 & 0.2 & 0.2 & 0.3 & 0.035 & ns & $* * *$ & ns & ns & ns \\
\hline Plastic & $0.1^{\mathrm{ab}}$ & $0.2^{\mathrm{ab}}$ & $0.3^{\mathrm{a}}$ & $0.2^{\mathrm{ab}}$ & $0.2^{\mathrm{ab}}$ & $0.2^{\mathrm{ab}}$ & $0.1^{\mathrm{ab}}$ & $0.0^{\mathrm{b}}$ & 0.006 & ns & ns & ns & $\bullet$ & $*$ \\
\hline Persistent & 6.3 & 5.9 & 6.2 & 6.0 & 5.5 & 5.0 & 5.4 & 5.4 & 0.085 & ns & $* * *$ & ns & ns & ns \\
\hline
\end{tabular}

${ }^{\text {a }}$ Values reported are the mean from triplicate cheese samples $(\mathrm{n}=3)$; values with different superscript letters differ at $P<0.05$ by statistical Benjamini-Hochberg test $(\bullet$

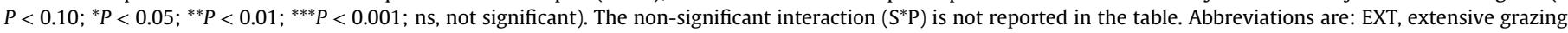
system; SEMI, semi-intensive grazing system; S, grazing system; T, treatment of milk; P, period; SEM, standard error of the means.

$\mathrm{R}$ milks were less elastic than those made in September, while no difference was observed in PA cheeses. In July, the R cheeses had a less "Swiss type cheese" odour than PA cheeses, while no difference was observed in cheeses made in September.

A significant effect of the grazing system used was observed only for six out of thirty-five sensory attributes, regardless of the milk treatment and the period of cheese-making. The quantity of spots on the rind was slightly lower, and the cheese cores were less yellow $(P<0.05)$ for EXT than for SEMI cheese rinds. The latter were creamier $(P<0.05)$ and more adhesive $(P<0.01)$. Moreover, EXT cheeses were slightly saltier and more sour $(P<0.05)$ than SEMI cheeses. No sensory attributes related to odour and aroma were affected by the grazing system used.

The $S^{*} \mathrm{~T}$ interaction affected few cheese sensory properties. Among PA cheeses, the EXT cheeses had greyer rinds than the SEMI cheeses, while no difference was observed between $\mathrm{R}$ cheeses from the two grazing systems. The SEMI cheeses were more elastic than EXT cheeses, in particular for the R cheeses. The "fermented cream" aroma was higher for EXT cheeses than SEMI cheeses in R cheeses, while no difference was observed among PA cheeses.
Concerning the period of cheese-making, the differences between cheeses made in July and in September concerned mainly the appearance of the cheese core, regardless of the grazing system and the milk treatment used. Cheeses made in September were judged as having a whiter $(+0.6, P<0.001)$ and a more marbled core $(+1.1, P<0.001)$ than July cheeses. The latter had a more intense taste $(P<0.05)$. The period had slight effects on the sensory attributes of odour, texture, and aroma.

\subsection{Relationships between sensory characteristics and the biochemical and microbial composition of Cantal-type cheeses}

PCA was performed to summarise the main relationships between sensorial, biochemical and microbial data accounting for effects attributable to the grazing system, milk treatment, and period of cheese-making used (Fig. 1). The first and second principal components accounted for $62 \%$ of the total variance. Generally, the sensory variables corresponding to aroma, odour, and taste were positively correlated (Fig. 1A). These variables were also positively correlated with the levels of microbial groups, 

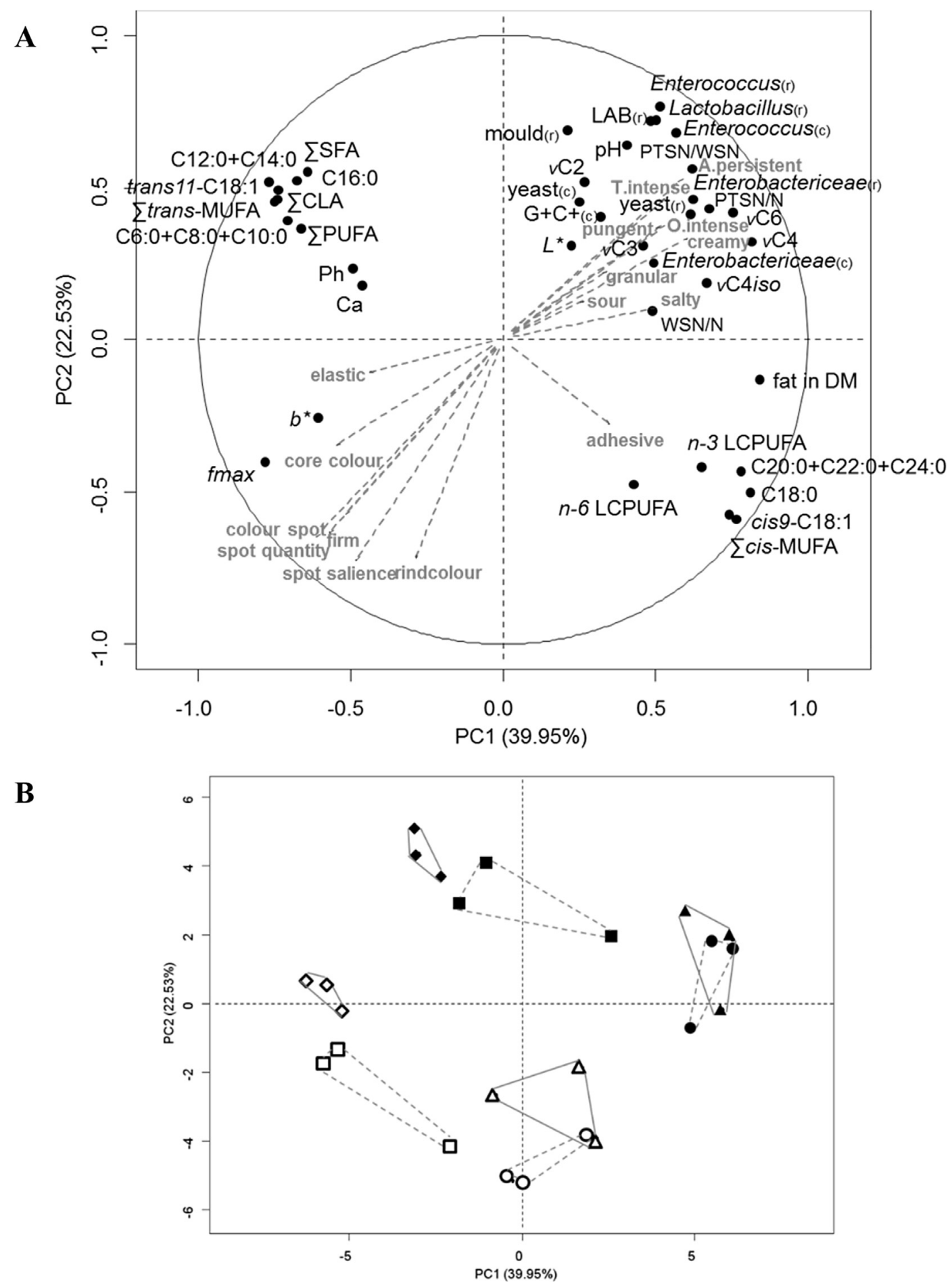

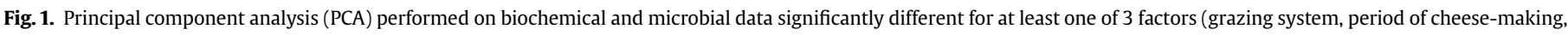

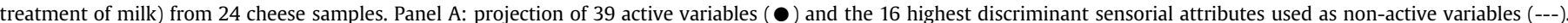

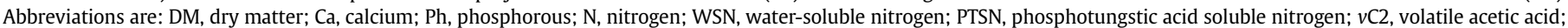

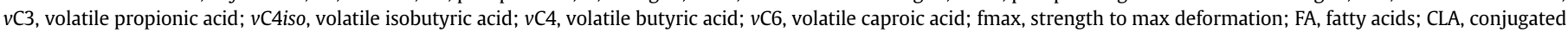

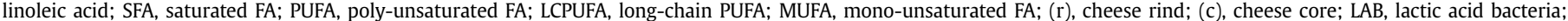

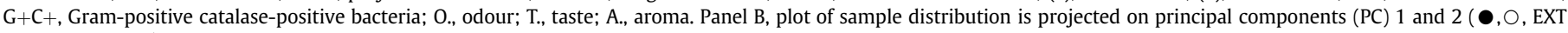

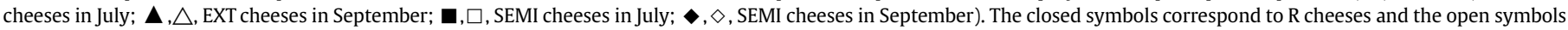
to PA cheeses. The dotted line corresponds to cheeses made in July and the continuous line to cheeses made in September. 
proteolysis, and VFA concentration. All these variables were negatively correlated with the sensory variables corresponding to firmness and rind appearance. No FA concentration was significantly correlated with the variables cited above. The sums of SFA, PUFA, and CLA were negatively correlated with those of LCPUFA and cis-MUFA. On the first axis, R cheeses were characterised by higher levels of microbial groups, higher VFA concentrations, and higher levels of proteolysis in relation to stronger odours and tastes and more persistent aromas than PA cheeses (Fig. 1B). PA cheeses had higher firmness and appearance estimators than $\mathrm{R}$ cheeses. On the second axis, EXT and SEMI cheeses were mainly differentiated by their FA profiles.

\section{Discussion}

\subsection{Grazing systems}

The originality of this study is that the same milking machine and the same facilities and conditions of cheese-making and ripening were used for both grazing systems, in contrast with most field studies, and thus grazing system is the major factor explaining potential differences in milk and cheese microbial and chemical compositions.

The main influence of grazing systems concerned cheese texture, which is known to vary according to cheese fat in DM, calcium and phosphorus contents, FA profile, and proteolysis (Fox, Law, McSweeney, \& Wallace, 1999). The higher fat in DM of EXT than SEMI cheeses is concordant with their creamier and more adhesive textures and is directly linked to the higher fat to protein ratio of the corresponding milks. The grazing diet in the EXT system is characterised by no concentrate distribution. The lower energetic density and the higher fibre content of the diet in EXT systems (Supplementary Table S1) reduced the protein content of the milk produced and increased the fat content of the milk, respectively. These observations were particularly more pronounced in September, when the EXT cows grazed on mature grass. Moreover, the energy expended by cows walking was higher in the EXT system, where the grazing surface was the largest. The higher body reserve mobilisation of EXT cows may contribute to the higher cis9$\mathrm{C} 18: 1$ content in their milk because cis9-C18:1 is the primary FA released from body reserves during their mobilisation (Kay et al., 2005). In contrast, the greater milk yield of SEMI cows could reflect the improved nutritional status of these cows, as previously reported by Pires et al. (2015).

Cheese FA profiles may also contribute to the differences found in cheese texture according to the grazing system. The influence of FA composition on cheese texture is mainly linked to C16:0 and cis9-C18:1 concentrations, which are the major milk saturated and monounsaturated FAs and have high and low melting points, respectively. In our study, the EXT cheeses (versus SEMI cheeses) were creamier, which is in agreement with their higher cis9-C18:1 to $\mathrm{C16:0}$ ratios (Martin et al., 2005). As previously reported by many authors (Bodas et al., 2010; Lucas et al., 2006), the FA profiles of cheeses reflect those of milks. The latter can be modulated by diet characteristics such as the amount of concentrate or the botanical composition of grassland pastures (Chilliard et al., 2007; Ferlay, Martin, Pradel, Coulon, \& Chilliard, 2006). Our results confirm that the concentrations in milk of short- and medium-chain FA and trans-C18:1 isomers are higher in SEMI than EXT milks, and the concentrations of cis9-C18:1 and $n-3$ LCPUFA are lower due to the combined effects of concentrate distributed to SEMI cows (Chilliard et al., 2007) and the lower botanical diversity of grasslands (Farruggia et al., 2014). These effects could be due partly to the leafier herbage grazed in the SEMI system. When herbage matures, the concentrations of trans11-C18:1 and cis9trans11-CLA decrease while that of C16:0 increases (Coppa et al., 2015). We can notice that in EXT milks and cheeses, the lower concentration of the major atherogenic FA (C12:0, C14:0, C16:0) combined to the higher concentration of cis9-C18:1 and C18: $3 n-3$ can be considered as an advantage from a nutritional point of view (Bodas et al., 2010; Gómez-Cortés et al., 2009). On the contrary, the higher content of trans11-C18:1 and cis9trans11-CLA in SEMI milks and cheeses may be considered as an advantage regarding their putative role in the prevention of cancer (Shingfield, Bernard, Leroux, \& Chilliard, 2010). However, the differences in milk FA profiles between the two grazing systems were limited because both diets were based essentially on grazed grass, as was previously shown by Coppa et al. (2011b).

The yellower cores of SEMI cheeses compared with EXT cheeses may be linked to a higher fat concentration of carotenoids in SEMI cheeses because these pigments are known to have a considerable effect on milk and cheese yellow colour (Nozière et al., 2006). Little or no change in the concentration of carotenoids between milks and cheeses was observed by these authors. The putative higher carotenoids concentration of SEMI milk fat is in accordance with the fact that, due to the higher stocking rate and rapid rotation among paddocks, the SEMI cows were allowed to graze herbage at leafy stage rich in carotenoids (Nozière et al., 2006), especially in September.

The cheeses from the two studied grazing systems were not clearly differentiated according to their flavours or their odours, even if EXT cheeses were slightly saltier and sourer than SEMI cheeses, in agreement with Coppa et al. (2011b). This result contrasts with studies reporting that the most diversified grasslands give intense and diversified aromas to cheeses (Coulon et al., 2004; Martin et al., 2005). Surprisingly, the cheese flavour seems unaffected by the more advanced secondary proteolysis and by the higher concentration of volatile butyric acid in EXT cheeses, which is usually associated to strong cheese flavour (Cornu et al., 2009). We can suppose that the quantitative differences of proteolysis or VFA concentrations observed between the grazing systems were too weak to be perceived by the panellists. Moreover, microbial activities play a key role in the development of cheese flavours (Montel et al., 2014). Although the levels of several microbial groups were higher in EXT than in SEMI milks, microbial levels in cheeses were weakly modified by the grazing system, except for yeast levels, which were higher in EXT cheese rinds. The same starter cultures were added to all cheeses for acidification and ripening, which may have impeded the development of the raw milk microbiota, thereby lessening the differences between grazing systems.

The high spot quantities observed in our study on SEMI cheese rinds were previously reported in Cantal-type cheeses manufactured with milks with high PUFA contents (Coppa et al., 2011b). Lerch et al. (2015) also observed differences in rind appearance in Saint-Nectaire type cheeses associated with Mucor (mould) development related to the FA composition of milk. Although the antimicrobial properties of FA and their derivatives have been known for many years (Kabara, Swieczkowski, Conley, \& Truant, 1972), their effects on cheese surface microbiota have not been evaluated (Monnet, Landaud, Bonnarme, \& Swennen, 2014). We can hypothesise that the FA profile of SEMI cheese rinds may confer an advantage to ripening starters that produce typical Cantal cheese spots, such as the mould S. casei (Coppa et al., 2011a), which could explain the observed differences in cheese rind appearance.

\subsection{Milk treatment}

The sensory properties of cheese were drastically modified by milk pasteurisation in our study. The more intense and diversified flavour of raw milk than pasteurised milk cheeses was previously 
reported by many authors using similar (Buchin et al., 1998; Cornu et al., 2009) or different cheese models (Bachmann et al., 2011; Montel et al., 2014; Van Hekken, 2012). The drop in the VFA concentrations in pasteurised milk cheeses observed in our study, and more generally the differences in the balance of volatile compounds as previously reported by Buchin et al. (1998) and Demarigny, Beuvier, Buchin, Pochet, and Grappin (1997) were closely related to the low levels and diversity of microorganisms in pasteurised milks and to the associated catabolic reactions in pasteurised milk cheeses. This may contribute to their more intense and diversified flavours as found in our study. An optimum flavour is achieved faster in raw milk cheeses due to the enhanced microbial activities in these cheeses (Bachmann et al., 2011; Grappin \& Beuvier, 1997). Cornu et al. (2009) suggested that softer odours such as "cream" and "fresh cream" in pasteurised milk Cantal-type cheeses would be a result of the decreased quantity of short-chain fatty acids released, which are odour-active compounds.

The more advanced proteolysis of raw milk cheeses made in July compared with pasteurised milk cheeses may explain their less elastic texture and their lower firmness as reported by Gaya, Medina, Rodriguez-Marin, and Nunez (1990). Proteolysis levels and the elasticity of pasteurised milk cheeses manufactured in July and September were similar, which confirms the negative association observed in PCA between proteolysis and cheese elasticity. Nevertheless, some authors found that proteolysis was lower in raw milk cheeses because of their lower moisture content (Grappin \& Beuvier, 1997), which we did not observe in this study.

Sensory analysis highlighted higher spot quantity and salience on the rinds of pasteurised milk cheeses compared with raw milk cheeses. The same ripening starters that contribute to the development of spots on Cantal cheeses rind were inoculated on the cloths of both the raw and the pasteurised milk cheeses. However, among the microbial groups potentially involved in ripening and comprising species added as ripening starters, the levels of Grampositive catalase-positive bacteria tended to be higher on the rinds of pasteurised milk cheeses, while the levels of yeasts and moulds were lower on the rinds of those cheeses. Although we do not know the precise levels of the ripening starter strains reached in each cheese, we can hypothesise that they grow more easily on the rinds of pasteurised cheeses because the levels of raw milk microbiota in these cheeses are reduced by pasteurisation.

In our trial, the cores of the pasteurised cheeses were considered the yellowest for every grazing system and cheese-making period studied. This effect of pasteurisation on cheese colour is infrequently mentioned in the literature, although it is a decisive factor for consumers when deciding to purchase cheeses. Despite the low temperature and the short duration of milk heating $\left(72^{\circ} \mathrm{C}, 20 \mathrm{~s}\right)$, we propose the hypothesis that this colouration could be linked to a non-enzymatic Maillard reaction (Van Boekel, 1998) caused by the interaction between reducing sugars and amino acids.

\subsection{The combined effect of grazing system and milk treatment}

Regarding cheese flavour, some authors have previously reported interactions between milk production conditions and milk heat treatment (Cornu et al., 2009). In particular, in cheeses derived from pasture or winter diets, Verdier-Metz et al. (2002) observed a higher effect of diet on cheese flavour when using raw milk than when using pasteurised milk. This suggests that this effect could be ascribed to milk components such as native enzymes from milk (e.g., LPL or plasmin) or milk microbiota that are modified by milk pasteurisation. In our study, the fermented cream aroma was more pronounced in EXT than in SEMI cheeses made from raw milk, whereas this effect was not observed with pasteurised milk cheeses. Similarly, differences in proteolysis and elasticity in raw milk cheeses between grazing systems were observed, while all pasteurised cheeses had similar characteristics. This difference in raw milk cheeses cannot be explained by milk LPL, because LPL did not vary in milk according to the grazing systems or the period of cheese-making. Levels of lactic bacteria (lactic acid bacteria and heterofermentative facultative Lactobacillus) differed in milk according to the grazing systems used, which could have affected the extent and nature of amino acids released by the degradation of peptides (action of microbial proteinases and peptidases) through proteolysis, and thus their transformation to catabolic products involved in cheese flavour (Kieronczyk, Skeie, Olsen, \& Langsrud, 2001; Marilley \& Casey, 2004). With regard to the level of microbial groups in ripened cheeses, only few interactions with milk treatment were observed. Where there were interactions, differences between periods of cheese-making were only observed in pasteurised milk cheeses. Therefore, the interactions between milk production conditions and milk heat treatment reported previously are only partly supported by our results. One explanation could be that the grazing systems used were too similar to exert a significant effect on the sensory properties of cheese.

\subsection{Period of cheese-making}

The effect of the cheese-making period on the sensory properties of cheese is weak in our study because the animal diet and cheese-making facilities were similar at both periods. Our result is contradictory to other studies (Agabriel et al., 2004; Esposito et al., 2014; Giaccone et al., 2016) where this effect was noteworthy because of the combination of different elements (season, feeding, management system).

\section{Conclusion}

In this experiment, we aimed to test whether the effects of farming systems on the sensory properties of cheese are partly eliminated when milk is pasteurised prior to cheese-making. This hypothesis was confirmed only for proteolysis, elasticity, and fermented cream aroma of cheeses. These differences could be associated to the different levels of lactic bacteria in raw milk of EXT and SEMI systems. Nevertheless, the two grazing systems were too similar to exert a significant effect on the sensory properties of cheeses. The effect of milk pasteurisation was the greatest when compared with other factors. The grazing systems tested resulted in important differences in milk FA profile, and hence in cheese texture. We noticed differences in the levels of several microbial groups in milks that were no longer present in the associated cheeses. The addition of the same starter cultures in all cheeses may explain that the cheese flavour was faintly affected. Although the effects of grazing systems on cheese rind appearance were not explained by the levels of microbial groups in cheese, we hypothesise that the grazing systems used may induce a modification in the balance of microbial species present and their associated microbial activities. Further research integrating a metabarcoding approach (sequencing of phylogenetic biomarker amplicons) will be needed to better understand the effects of the factors tested.

\section{Acknowledgements}

Financial support for this study was provided by the Auvergne region and FEDER funds in the framework of a CPER T2ANSH 2013 project $N^{\circ} 2101088272$ SyEL-inAuv (Systèmes d'Elevage laitier innovants en Auvergne. The authors wish to thank the staff of the INRA farm at Marcenat for animal care, René Lavigne for cheesemaking, Béatrice Desserre for microbiological analyses, Isabelle Constant for her valuable help in the analyses, Emilie Tixier for milk 
fatty acid analyses, Solange Buchin for volatile fatty acid analyses and Hélène Albouy for the sensory evaluation of the cheeses (ENILV Aurillac). The authors also thank the American Journal Experts for English proofreading.

\section{Appendix A. Supplementary data}

Supplementary data related to this article can be found at http:// dx.doi.org/10.1016/j.idairyj.2016.09.007.

\section{References}

Agabriel, C., Martin, B., Sibra, C., Bonnefoy, J. C., Montel, M. C., Didienne, R., et al. (2004). Effect of dairy production systems on the sensory characteristics of cantal cheeses: A plant-scale study. Animal Research, 53, 221-234.

Ardö, Y. (1999). Evaluating proteolysis by analysing the N content of cheese fractions. Bulletin-FIL-IDF, 337, 4-9.

Bachmann, H. P., Frohlich-Wyder, M. T., Jakob, E., Roth, E., Wechsler, D., Beuvier, E., et al. (2011). Raw milk cheeses. In J. W. Fuquay, P. F. Fox, \& P. L. H. McSweeney (Eds.), Encyclopedia of dairy sciences (2nd ed., Vol. 1, pp. 652-660). San Diego, CA, USA: Academic Press.

Bargo, F., Delahoy, J. E., Schroeder, G. F., \& Muller, L. D. (2006). Milk fatty acid composition of dairy cows grazing at two pasture allowances and supplemented with different levels and sources of concentrate. Animal Feed Science and Technology, 125, 17-31.

Bodas, R., Manso, T. Mantecón, A. R. Juárez, M., de la Fuente, M. A., \& GómezCortés, P. (2010). Comparison of the fatty acid profiles in cheeses from ewes fed diets supplemented with different plant oils. Journal of Agricultural and Food Chemistry, 58, 10493-10502.

Bovolenta, S., Romanzin, A., Corazzin, M., Spanghero, M., Aprea, E., Gasperi, F., et al. (2014). Volatile compounds and sensory properties of Montasio cheese made from the milk of Simmental cows grazing on alpine pastures. Journal of Dairy Science, 97, 7373-7385.

Buchin, S., Delague, V., Duboz, G., Berdague, J. L., Beuvier, E., Pochet, S., et al. (1998). Influence of pasteurization and fat composition of milk on the volatile compounds and flavor characteristics of semi-hard cheese. Journal of Dairy Science, 81, 3097-3108.

Bugaud, C., Buchin, S., Hauwuy, A., \& Coulon, J. B. (2001). Relationships between flavour and chemical composition of abondance cheese derived from different types of pastures. Lait, 81, 757-773.

Callon, C., Berdagué, J. L., Dufour, E., \& Montel, M. C. (2005). The effect of raw milk microbial flora on the sensory characteristics of Salers-type cheeses. Journal of Dairy Science, 88, 3840-3850.

Chilliard, Y., Glasser, F., Ferlay, A., Bernard, L., Rouel, J., \& Doreau, M. (2007). Diet, rumen biohydrogenation and nutritional quality of cow and goat milk fat. European Journal of Lipid Science and Technology, 109, 828-855.

Collomb, M., Bütikofer, U., Siber, R., Jeangros, B., \& Bosset, J. O. (2002). Composition of fatty acids in cow's milk fat produced in the lowlands, mountains and highlands of Switzerland using high-resolution gas chromatography. International Dairy Journal, 12, 649-659.

Coppa, M., Ferlay, A., Borreani, G., Revello-Chion, A., Tabacco, E., Tornambé, G., et al. (2015). Effect of phenological stage and proportion of fresh herbage in cow diets on milk fatty acid composition. Animal Feed Science and Technology, 208, 66-78.

Coppa, M., Ferlay, A., Monsallier, F., Verdier-Metz, I., Pradel, P., Didienne, R., et al. (2011a). Milk fatty acid composition and cheese texture and appearance from cows fed hay or different grazing systems on upland pastures. Journal of Dairy Science, 94, 1132-1145.

Coppa, M., Verdier-Metz, I., Ferlay, A., Pradel, P., Didienne, R., Farruggia, A., et al. (2011b). Effect of different grazing systems on upland pastures compared with hay diet on cheese sensory properties evaluated at different ripening times. International Dairy Journal, 21, 815-822.

Cornu, A., Rabiau, N., Kondjoyan, N., Verdier-Metz, I., Pradel, P., Tournayre, P., et al. (2009). Odour-active compound profiles in Cantal-type cheese: Effect of cow diet, milk pasteurization and cheese ripening. International Dairy Journal, 19, 588-594.

Coulon, J. B., Delacroix-Buchet, A., Martin, B., \& Pirisi, A. (2004). Relationships between ruminant management and sensory characteristics of cheeses: A review. Lait, 84, 221-241.

De Man, J. C., Rogosa, M., \& Sharpe, E. M. (1960). A medium for the cultivation of lactobacilli. Journal of Applied Microbiology, 23, 130-135.

Demarigny, Y., Beuvier, E., Buchin, S., Pochet, S., \& Grappin, R. (1997). Influence of raw milk microflora on the characteristics of Swiss-type cheeses. 2. Biochemical and sensory characteristics. Lait, 77, 151-167.

Denis, C., Gueguen, M., Henry, E., \& Levert, D. (2001). New media for the numeration of cheese surface bacteria. Lait, 81, 365-379.

Elgersma, A., Tamminga, S., \& Ellen, G. (2006). Modifying milk composition through forage. Animal Feed Science and Technology, 131, 207-225.

Esposito, G., Masucci, F., Napolitano, F., Braghieri, A., Romano, R., Manzo, N., et al. (2014). Fatty acid and sensory profiles of Caciocavallo cheese as affected by management system. Journal of Dairy Science, 97, 918-1928.
Farruggia, A., Pomiès, D., Coppa, M., Ferlay, A., Verdier-Metz, I., Le Morvan, A., et al (2014). Animal performances, pasture biodiversity and dairy product quality: How it works in contrasted mountain grazing system. Agriculture, Ecosystems and Environment, 185, 231-244.

Faulconnier, Y., Thévenet, M., Fléchet, J., \& Chilliard, Y. (1994). Lipoprotein lipase and metabolic activities in incubated bovine adipose tissue explants: Effects of insulin, dexamethasone, and fetal bovine serum. Journal of Animal Science, 72, 184-191.

Ferlay, A., Martin, B., Pradel, P., Coulon, J. B., \& Chilliard, Y. (2006). Influence of grass-based diets on milk fatty acid composition and milk lipolytic system in Tarentaise and Montbéliarde cow breeds. Journal of Dairy Science, 89, 4026-4041.

Fox, P. F., Law, J., McSweeney, P. L. H., \& Wallace, J. (1999). Biochemistry of cheese ripening. In P. F. Fox (Ed.), Cheese: Chemistry, physics and microbiology (pp. 389-438). Gaithersburg, MO, USA: Aspen Publishers Inc.

Gaya, P., Medina, M., Rodriguez-Marin, M. A., \& Nunez, M. (1990). Accelerated ripening of ewes' milk manchego cheese: The effect of elevated ripening temperatures. Journal of Dairy Science, 73, 26-32.

Giaccone, D., Revello-Chion, A., Galassi, L., Bianchi, P., Battelli, G., Coppa, M., et al. (2016). Effect of milk thermisation and farming system on cheese sensory profile and fatty acid composition. International Dairy Journal, 59, 10-19.

Gómez-Cortés, P., Frutos, P., Mantecón, A. R., Juárez, M., de la Fuente, M. A., \& Hervás, G. (2009). Effect of supplementation of grazing dairy ewes with a cereal concentrate on animal performance and milk fatty acid profile. Journal of Dairy Science, 92, 3964-3972.

Gomri, G. (1946). Buffers in the range of pH 6.5 to 9.6. Proceedings of Society for Experimental Biology and Medicine, 6, 233-234.

Grappin, R., \& Beuvier, E. (1997). Possible implications of milk pasteurization on the manufacture and sensory quality of ripened cheese. International Dairy Journal, 7, 751-761.

IDF. (1990). Milk and milk products. Enumeration of microorganisms (colony count technique at $30^{\circ} \mathrm{C}$ ). IDF standard $100 \mathrm{~B}$. Brussels, Belgium: International Dairy Federation.

Irlinger, F., \& Mounier, J. (2009). Microbial interactions in cheese: Implications for cheese quality and safety. Current Opinion in Biotechnology, 20, 142-148.

Isolini, D., Grand, M., \& Glättli, H. (1990). Selective medium for the detection of obligatory and facultative hetefermentative lactobacilli. Schweizerische Milchwirtschaftliche Forschung, 19, 57-59.

Jellema, A., Anderson, M., Heeschen, W., Kuzdal-Savoie, S., Needs, E. C., Suhren, G. et al. (1991). Determination of free fatty acids in milk and milk products. IDF bulletin $N^{\circ} 265$. Brussels, Belgium: International Dairy Federation.

Kabara, J. J., Swieczkowski, D. M., Conley, A. J., \& Truant, J. P. (1972). Fatty acids and derivatives as antimicrobial agents. Antimicrobial Agents and Chemotherapy, 2 $23-28$.

Kay, J. K., Weber, W. J., Moore, C. E., Bauman, D. E., Hansen, L. B., Chester-Jones, H., et al. (2005). Effects of week of lactation and genetic selection for milk yield on milk fatty acid composition in Holstein cows. Journal of Dairy Science, 88, 3886-3893.

Kieronczyk, A., Skeie, S., Olsen, K., \& Langsrud, T. (2001). Metabolism of amino acids by resting cells of non-starter lactobacilli in relation to flavour development in cheese. International Dairy Journal, 11, 217-224.

Lerch, S., Ferlay, A., Graulet, B., Cirié, C., Verdier-Metz, I., Montel, M. C., et al. (2015) Extruded linseeds, vitamin $\mathrm{E}$ and plant extracts in corn silage-based diets of dairy cows: Effects on sensory properties of raw milk and uncooked pressed cheese. International Dairy Journal, 51, 65-74.

Lucas, A., Rock, E., Chamba, J. F., Verdier-Metz, I., Brachet, P., \& Coulon, J. B. (2006) Respective effects of milk composition and the cheese-making process on cheese compositional variability in components of nutritional interest. Lait, 86, $21-41$.

Marilley, L., \& Casey, M. G. (2004). Flavours of cheese products: Metabolic pathways, analytical tools and identification of producing strains. International Journal of Food Microbiology, 90, 139-159.

Martin, B., Pomiès, D., Pradel, P., Verdier-Metz, I., \& Rémond, B. (2009). Yield and sensory properties of cheese made with milk from Holstein or Montbéliarde cows milked twice or once daily. Journal of Dairy Science, 92, 4730-4737.

Martin, B., Verdier-Metz, I., Buchin, S., Hurtaud, C., \& Coulon, J. B. (2005). How do the nature of forages and pasture diversity influence the sensory quality of dairy livestock products? Animal Science, 81, 205-212.

Mayeux, J. V., Sandine, W. E., \& Elliker, P. R. (1962). A selective medium for detecting Leuconostoc in mixed-strain starter cultures. Journal of Dairy Science, 45, 655-661.

Monnet, C., Landaud, S., Bonnarme, P., \& Swennen, D. (2014). Growth and adaptation of microorganisms on the cheese surface. FEMS Microbiology Letters, 263, $1-9$.

Montel, M. C., Buchin, S., Mallet, A., Delbès-Paus, C., Vuitton, D. A., Desmasures, N., et al. (2014). Traditional cheeses: Rich and diverse microbiota with associated benefits. International Journal of Food Microbiology, 177, 136-154.

Mossel, D. A. A., Mengerink, W. H. J., \& Scholts, H. H. (1962). Use of a modified MacConkey agar medium for selective growth and enumeration of all Enterobacteriaceae. Journal of Bacteriology, 84, 381-384.

Mossel, D. A. A., Visser, M., \& Merrgerinsk, W. H. J. (1962). A comparison of media for the enumeration of moulds and yeasts in food and beverages. Laboratory, 11 109-112. 
Nozière, P., Graulet, B., Lucas, A., Martin, B., Grolier, P., \& Doreau, M. (2006). Carotenoids for ruminans: From forages to dairy products. Animal Feed Science and Technology, 131, 418-450.

Pires, J. A. A., Chilliard, Y., Delavaud, C., Rouel, J., Pomiès, D., \& Blanc, F. (2015) Physiological adaptations and ovarian cyclicity of Holstein and Montbéliarde cows under two low-input production systems. Animal, 9, 1986-1995.

Shingfield, K. J., Bernard, L., Leroux, C., \& Chilliard, Y. (2010). Role of trans fatty acids in the nutritional regulation of mammary lipogenesis in ruminants. Animal, 4 , $1140-1166$.

Slanetz, L. C. W., \& Bartley, C. H. (1957). Numbers of enterococci in water, sewage and feces determinated by the membrane filter technique with an improved medium. Journal of Bacteriology, 74, 591-596.
Van Boekel, M. A. (1998). Effect of heating on Maillard reactions in milk. Food Chemistry, 62, 403-414.

Van Hekken, D. (2012). Quality aspects of raw milk cheeses. Food Technology, 66, 66-78.

Verdier-Metz, I., Coulon, J. B., Pradel, P., Viallon, C., Albouy, H., \& Berdagué, J. L. (2000). Effect of botanical composition of hay and casein genetic variants on the chemical and sensory characteristics of ripened Saint-Nectaire type cheese. Lait, $80,360-370$.

Verdier-Metz, I., Martin, B., Hulin, S., Ferlay, A., Pradel, P., \& Coulon, J. B. (2002) Combined influence of cow diet and pasteurisation of milk on sensory properties of French PDO Cantal cheese. In CIDIL (Ed.), Proceedings of Congrilait 26th IDF world dairy congress. Paris, France: CDR. 